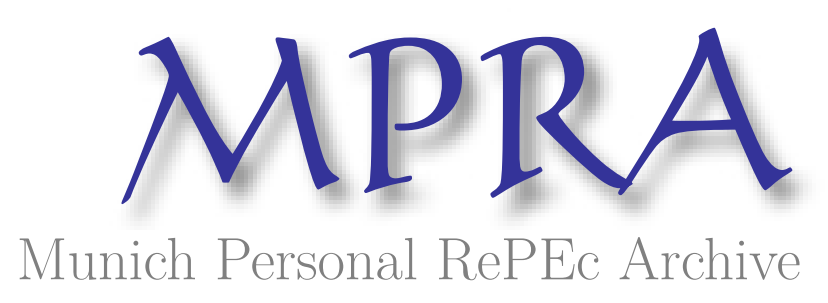

\title{
Income Taxation when Markets are Incomplete
}

Tirelli, Mario

20 December 2002

Online at https://mpra.ub.uni-muenchen.de/746/

MPRA Paper No. 746, posted 09 Nov 2006 UTC 


\title{
Income taxation when markets are incomplete
}

\author{
Mario Tirelli \\ Department of Economics, Università di Roma Tre \\ e-mail: tirelli@uniroma3.it
}

Received: 19 October 2001 / Accepted: 19 December 2002 - (C) Springer-Verlag 2003

\begin{abstract}
We investigate the welfare effects of proportional income taxation in a standard general equilibrium model with incomplete markets (GEI). Formally, our analysis is on the allocative effects of state-contingent income tax reforms. Tax reforms are restricted to be anonymous, publicly and truthfully announced before markets open, and they are required to result in an ex-post constrained efficient allocation. Our main result is to show that there do typically exist contingent tax reforms that are Pareto improving. These reforms, acting directly on the asset span, modify private risk-sharing opportunities. Thus, unlike most of the GEI literature, the type of policy transmission mechanism considered does not rely on second-order, relative spot price effects. Yet, the key welfare effects of our tax reforms are substantially equivalent to those induced through changes in relative spot prices, as, for example, in Geanakoplos and Polemarchakis (1986), Geanakoplos et al. (1990), or in Citanna et al. (2001).
\end{abstract}

Mathematics Subject Classification (2000): 58E17, 46N10, 93B29

Journal of Economic Literature Classification: D52, H21, H24, H25

I am particularly grateful to Paolo Siconolfi who advised me on earlier drafts of this paper. I thank J. Drèze, M. Marchand, H. Polemarchakis, M. Wooders, and the participants of the economic theory seminars at CORE, University of Pennsylvania, and Columbia University, for comments. I also thank two anonymous referees of this journal for their very accurate job. Remaining errors are my own. Financial support from the Franqui Foundation and the European $T M R$ program is gratefully acknowledged.

This version of the paper was prepared while I was a visiting professor at CORE-UCL. 


\section{Introduction}

The use of state-contingent taxation has always been hotly debated. Besides counter-cyclical active fiscal policies of Keynesian tradition, policy makers have been concerned with the problem of designing and implementing passive, state-contingent tax rules. In this paper we investigate the welfare effects of linear income taxation in economies in which consumers cannot fully efficiently diversify economic risk. In a standard, two periods, general equilibrium model, with incomplete markets (GEI), we explore the effects of state-contingent income tax reforms on individual risk sharing. Our main result is to show that there do, typically, exist tax reforms that result in Pareto superior equilibria (see Theorem 1). Moreover, we show that this is true both in a pure exchange GEI, and in a production GEI with stock markets. Finally, we also argue that tax reforms may have welfare effects of the opposite sign (i.e., tax reforms may effectively change individual utility levels in any direction; see Corollary 1); this latter feature comes as a warning to policy makers who are called upon to design this type of reform.

Formally, our welfare analysis follows the general prescriptions of the public finance literature on (gradual) policy reforms (see, for example, Guesnerie (1977), and Feldstein (1976)). Given an initial economy, we ask if there exists a feasible direction of tax changes such that the initial equilibrium allocation can be locally Pareto improved. We then prove that such tax reforms are robust, by showing that their effects hold generically, that is, for an open and dense subset of economies parameterized by endowments, preferences, and production technologies.

The class of tax reforms we analyze has the following features:

- reforms take the underlying market structure as given;

- they are ex-post constrained Pareto optima;

- they are anonymous;

- they are restricted to achieve state-by-state budget balance.

A local marginal change of the tax system acts directly on the asset span, changing (but not augmenting) consumers' ability to redistribute income across contingencies; precisely, tax reforms do not change the degree of market incompleteness. Re-allocations are completely decentralized: after a tax reform a new equilibrium allocation is achieved competitively, through individual trade on the existent markets. We then say that our tax reforms are (not only ex-ante but also) ex-post constrained Pareto optima. Moreover, the type of taxation considered is anonymous. Finally, state-by-state budget balance inhibits the planner to implementing across states- (and time-) income redistributions directly. 
The original aspect of our result is the effectiveness of contingent tax reforms in modifying consumers' risk-sharing opportunities, through a change in the asset span position. The highly simplified representation of our economy and of the central government, aims precisely to isolate this effect, and, ultimately, to derive a minimal set of conditions under which this type of taxation leads to well identified welfare effects. Thus, for example, we assume that a single perishable commodity is traded in the economy. In contrast with most of the GEI literature, our results are not driven by changes in relative spot prices (see, for example, Geanakoplos and Polemarchakis (1986), Geanakoplos et al. (1990), Citanna et al. (1998, 2001)). Asset span variations are powerful enough to generate changes in individual utilities that are otherwise typical of spot price, second-order effects (see Section 3.2, Remark 2).

The transmission mechanism of tax reform is also different from the one characterizing financial innovation in the sense of Cass and Citanna (1998) and of Elul (1995, 1999). Precisely, in contrast with Elul (1999), we assume that the number of assets (or asset markets) is given, and cannot be modified by the central government. Thus, although tax reforms may tilt the asset span, they do not change its dimension.

Our representation of the central government is also highly simplified. A fictitious central planner set ad valorem, anonymous, state-contingent taxes (and/or subsidies) on individuals' incomes, subject to a state-by-state fiscal budget constraint. Less restrictive budgetary rules and/or other typical motives for public intervention, if introduced, would only strengthen our results. Similarly, we could, and indeed we do, discuss the case in which income may be taxed at different rates, depending on its source (endowment income, returns from different assets, etc.). A necessary condition for our results to hold is that the number of policy objectives does not exceed the number of tax instruments: the changes of the utility levels corresponding to the $H$ consumer types must be less than the tax instruments. This condition is an incomplete market analogue of that due to Tinbergen (1952).

The approach used to prove our results is comparative statics, and relies on well-known techniques of differential topology. In particular, the welfare analysis follows the guidelines traced in Smale (1974), later applied and extended by Geanakoplos and Polemarchakis (1986) and, more recently, by Citanna et al. (1998), whose underlying approach is used in this paper.

Some concluding observations may help the reader. First, our contingent taxes are not progressive in income. They are linear income taxes, such as those typically used to tax individual income from bond holdings. The assumption that tax reforms may be state-contingent is realistic inasmuch as it is the decision of implementing a tax scheme contingently on factors exogenous to the underlined model of the economy, factors which may be 
accounted for in the definition of the state space (e.g., aggregate supply shocks, financial crises, etc.). Secondly, our model does not endogenously provide an explanation of why markets are incomplete. Extensions in this direction, although potentially interesting, are beyond the scope of this paper. Moreover, at this stage, our positive analysis of tax reform does not provide any specific recipe for policy intervention. Yet, our results do indicate and analyze interesting effects that income taxes may exhibit in incomplete market economies. Finally, our analysis is local. We do not derive an optimal fiscal policy rule: a mapping from the equilibrium set to the set of feasible tax rates which a central government should optimally enforce. We instead trace the direction of (robust) welfare-improving tax reforms.

Our work is organized as follows. In Section 2 we present the benchmark economy, and give a notion of competitive equilibrium. In Section 3, we state and discuss our main results (Theorem 1 and Corollary 1). There, we also discuss and compare our notion of constrained optimality with the standard ones used in the GEI literature; this is done by mean of a few examples, both for a pure exchange and for a production economy. The proofs of our results are in Section 4, which also includes other technical results such as the (local) existence and regularity of equilibria (Theorem 2 and Corollary 2).

\section{The model}

\subsection{The general framework}

2.1.1. Private agents and commodities There are two dates indexed by 0 and 1 . At date 1 there is a finite number $S$ of possible states of the world, $s=1, \ldots, S$, and $\mathcal{S}=\{1, \ldots, S\}$. We also use the convention of labeling date 0 as $s=0$. There is a single perishable good that can be used both for consumption and investment at date 0 , and for consumption at date 1 . We denote by $N=S+1$ the number of contingent goods in the economy.

There are $H \geq 2$ consumers, $\mathcal{H}=\{1, \ldots, h, \ldots, H\}$. Every consumer $h$ is endowed with a vector $\left(e_{0}^{h}, e_{1}^{h}\right) \in \mathbb{R}_{++}^{N}$ of contingent goods, where $e_{1}^{h}=\left(e_{1}^{h}, \ldots, e_{S}^{h}\right)$ denotes date 1 endowments. For simplicity, we assume that each individual consumption set coincides with the nonnegative orthant of the commodity space, $\mathbb{R}_{+}^{N}$, and we denote a typical element by $\left(x_{0}, x_{1}\right)$. The utility function $u^{h}: \mathbb{R}_{+}^{N} \rightarrow \mathbb{R}$ represents the preference ordering of consumer $h$ over his consumption set. Lastly, some rather strong, but standard, assumptions on preferences and endowments are introduced.

Assumption 1. For every $h$ in $\mathcal{H}$ :

(1) $u^{h}$ is twice continuously differentiable, and differentiably strictly increasing $\left(D u^{h}(x) \gg 0 \forall x \in \mathbb{R}_{++}^{N}\right)^{1}$; 
(2) $u^{h}$ is differentiably strictly quasi-concave $\left(r D^{2} u^{h}(x) r^{T}<0 \forall r \in \mathbb{R}^{N}\right.$, $r \neq 0$, such that $\left.D u^{h}(x) r^{T}=0 \forall x \in \mathbb{R}_{++}^{N}\right)$;

(3) indifference surfaces are contained in the interior of the positive orthant (the closure of $\left\{x \in \mathbb{R}_{++}^{N}: u^{h}(x) \geq u^{h}\left(x^{\prime}\right)\right\}$ is contained in $\mathbb{R}_{++}^{N} \forall x^{\prime} \in$ $\mathbb{R}_{++}^{N}$ );

(4) endowments are strictly positive $\left(e^{h} \gg 0\right)$.

We denote by $U$ the set of utility functions satisfying Assumption 1(1) through 1(4).

There are $J \geq 1$ assets, $\mathcal{J}=\{1, \ldots, j, \ldots, J\}$. Assets may be characterized either by fixed return (e.g., bonds) or by returns that depend on endogenous variables (e.g., stocks or equities). Precisely, we let $\mathcal{J}=\mathcal{J}_{1} \cup \mathcal{J}_{2}$, and $J=J_{1}+J_{2}$, where $\mathcal{J}_{1}$ is the set of $J_{1}$ assets with fixed return matrix $R \in \mathbb{R}_{+}^{N J_{1}}$ (say bonds), possibly empty, and $\mathcal{J}_{2}$ is the set of securities whose return matrix is endogenous and depends on production activities (say stocks). Pure exchange economies, with an empty set $\mathcal{J}_{2}$, will also be considered in the paper.

Production is carried out by $J_{2}$ competitive firms. The technology of a typical firm $j$ in $\mathcal{J}_{2}$ is represented by its production possibility set $Y^{j} \subset \mathbb{R}^{N}$, with typical input-output vector $y^{j}=\left(y_{0}^{j}, y_{1}^{j}\right)$. Further, firms are endowed with a vector $\eta^{j}$ in $\mathbb{R}_{++}^{N}$ of contingent goods. The properties of the production technologies are summarized in the following.

Assumption 2. For every firm $j$ in $\mathcal{J}_{2}$ :

(1) $Y^{j} \subset \mathbb{R}^{N}$ is closed and convex, $0 \in Y^{j}, Y^{j} \cap \mathbb{R}_{+}^{N}=\{0\}$ and $\mathbb{R}_{-}^{N} \subset Y^{j}$;

(2) $\left(\sum_{h} e^{h}+\sum_{j}\left(Y^{j}+\eta^{j}\right) \cap \mathbb{R}_{+}^{N}\right)$ is compact, and $\forall h, e^{h} \in \mathbb{R}_{++}^{N}$;

(3) let $\mathcal{M}^{j} \subset \mathbb{R}^{N}$ be a $m_{j}$-dimensional subspace with $1 \leq m_{j} \leq N$; then $Y^{j} \subset \mathcal{M}^{j}$ is an $m_{j}$-dimensional manifold with boundary; its boundary, $\partial Y^{j}$, is twice continuously differentiable, and differentiably strictly quasi-convex at each point;

(4) firms' endowments are strictly positive $\left(\eta^{j} \gg 0\right)$.

Further, we assume that the technology set $Y^{j}$ can be represented by $Y^{j}=\left\{y_{j} \in \mathbb{R}^{N}: f^{j}\left(y^{j}+\eta^{j}\right) \leq 0, \eta^{j} \in \mathbb{R}_{++}^{N}\right\}$, where $f^{j}: \mathbb{R}^{N} \rightarrow \mathbb{R}$ is a differentiable transformation function. We also assume that $\left(-y_{0}^{j}, y_{1}^{j}\right)>0$, and denote the typical element of $Y^{j}$ by $y=\left(y_{0}^{j}, y_{1}^{j}\right)$.

Assumption 2 implies that $f^{j}(\cdot)$ is twice continuously differentiable, non-decreasing and strictly quasi-convex, and that it satisfies $f^{j}\left(\eta^{j}\right)=0$. We denote by $\mathrm{Y}$ the set of transformation functions satisfying Assumption 2(1) through 2(3). Finally, for notational simplicity, in the rest of the paper we simply write $y^{j}$ for $y^{j}+\eta^{j}$. Firms' endowments $\eta^{j}$ are only needed

1 Hereafter, we use the convention that $>$, when applied to vectors, denotes a weak inequality ( $\gg$ a strong inequality). 
to prove the existence of equilibria, and do not play any role in our welfare analysis of tax reforms.

2.1.2. Government, income taxes, and fiscal budget rule We define a tax policy by specifying a set of tax variables and a budgetary rule. Further, as usual, we call instruments those policy variables that are independently controlled by the central planner.

We consider a tax policy in which, at date 0 , the planner announces a system of ad valorem taxes (and/or subsidies, or allowances) on consumers', date 1, personal income. This system of taxes and allowances makes individual taxation a non-linear function of income: different levels of statecontingent personal income may be taxed/subsidized at different rates. As we shall make clear as we go along, the type of taxation that produces interesting welfare effects in our simplified setting is one that modifies the state-contingent profile of the returns from portfolio holdings. For this reason, hereafter, we shall focus on "capital income taxation". State-contingent, anonymous, lump-sum taxes/subsidies will only be introduced as a way of achieving fiscal budget balance state-by-state: when capital income in state $s$ is taxed, its total revenue is redistributed lump-sum in the same state $s$.

The following alternative policy schemes, if introduced, would leave the rest of the analysis and results substantially unchanged:

(a) a proportional, state-contingent, anonymous tax/subsidy system on individual total income (returns from portfolio holdings plus endowment income);

(b) a proportional, state-contingent, asset (or sector) specific tax/subsidy system on individual capital income.

In (b) we refer to the case in which bonds and equities can be taxed at one or more different rates. Moreover, for bonds, (b) accounts for the case in which interest payments are tax deductible, as well as for the case in which they are not.

More formally, a portfolio $\theta_{j}^{h}$ of a stock $j \in \mathcal{J}_{2}$ yields to $h$ a before-tax return $\theta_{j}^{h} y_{s}^{j}$ in state $s$ for all $s$ in $\mathcal{S}$. Similarly, a portfolio $\theta_{j}^{h}$ of a bond $j \in \mathcal{J}_{1}$ yields to $h, \theta_{j}^{h} R_{s}^{j}$, in $s$ for all $s$ in $\mathcal{S}$. Let $T_{s}^{1}$ be an open bounded subset of $\mathbb{R}$ with typical element $t_{s}^{1} \neq-1$, with $T^{1}=\prod_{s=1}^{S} T_{s}^{1}$, ${ }^{2}$ and denote the set of feasible state-contingent taxes (subsidies) by $\mathcal{T}=\mathbb{R}^{S} \times T^{1}$. We formalize the following general fiscal budget rule.

2 An alternative way of defining of the tax domain is to assume that $T^{1}$ is the interior of a unit $(S-1)$-sphere. This would still be without loss of generality, since our analysis is entirely local, around the zero vector of policy instruments. 
Definition 1 (Fiscal budget balance). For every production plan $y \in Y$, and portfolio $\theta \in \mathbb{R}_{+}^{J H}$,

$$
\sum_{h \in \mathcal{H}}\left(t_{s}^{0}+t_{s}^{1}\left(\sum_{j \in \mathcal{J}_{1}} \theta_{j}^{h} R_{s}^{j}+\sum_{j \in \mathcal{J}_{2}} \theta_{j}^{h} y_{s}^{j}\right)\right)=0
$$

for all $\left(t_{s}^{0}, t_{s}^{1}\right)=t_{s} \in \mathcal{T}_{1, s}$ and all $s \in \mathcal{S}$.

Our assumption defines the government budget constraint, imposing fiscal budget balance state-by-state. This implies that no centralized transfers across time or states of the world are allowed. Moreover, in every state $s$, one tax variable is not a policy instrument; precisely, if, in state $s, t_{s}^{1}$ is controlled by the central government, $t_{s}^{0}$ is endogenously determined by the fiscal budget constraint. The choice of policy instruments, between $t^{0}$ and $t^{1}$, is completely arbitrary, it may be different across states, and it does not affect any of the latter results. Finally, two possible tax regimes can be considered:

(i) one in which interest payments are tax-deductible at the rate $t^{1}$;

(ii) a system in which interest earnings are taxed, but interest payments are not tax deductible.

In the first system, say, a tax rate $t_{s}^{1}<0$ is imposed on the return $R_{s}^{j} \theta_{j}$ from holding a bond portfolio $\theta_{j}$, in state $s$; then, the net interest paid to the bond holders by the bond issuer is $\left(1+t_{s}^{1}\right) R_{s}^{j} \theta_{j}$ (i.e., bond $j$ interest payments are tax deductible). This conforms to Definition 1. In a system of type (ii), with no tax deductible interest payments, the fiscal budget constraint is $H t_{s}^{0}+t_{s}^{1}\left(\sum_{j \in \mathcal{J}_{1}} \max \left(\theta_{j}^{h}, 0\right) R_{s}^{j}+\sum_{j \in \mathcal{J}_{2}} y_{s}^{j}\right)=0$.

Let $\mathcal{T} \subset \mathbb{R}^{2 S}$ be the set of taxes, with typical element $t=\left(t^{0}, t^{1}\right)$. Hereafter, to ease the exposition, we denote by $t^{I}$ the $S$-vector of policy instruments, and by $T^{I}(\subset \mathcal{T})$ its domain.

2.1.3. Spot and security markets There are $N(=(S+1))$ spot markets in the economy. Since the only good produced is perishable, consumers' saving can only take place through portfolio holdings (i.e., financial markets fully specify the available saving technology).

Every consumer, $h$, holds an initial ownership share (or portfolio endowment) $\bar{\theta}_{j}^{h} \in[0,1]$ such that $\sum_{h} \bar{\theta}_{j}^{h}=1$ for all firms $j$ in $\mathcal{J}_{2}$. At date 0 , when financial markets open, $h$ can trade for a final portfolio vector $\theta^{h}$. Further, since in our simple economy ownership implies control, we assume no-short sales, $\theta_{j}^{h} \geq 0$. The market price of a stock $j$ in $\mathcal{J}_{2}$ is $q^{j}$. When $h$ purchases $\theta_{j}^{h}$ stocks in firm $j$, she pays $q^{j} \theta_{j}^{h}$ and participates in the firm's 
investment by a corresponding $y_{0}^{j} \theta_{j}^{h}$, at date 0 , and $\theta_{j}^{h}$ entitles $h$ to receive $y_{s}^{j} \theta_{j}^{h}$ in state $s$ (for all $s$ in $\mathcal{S}$ ), at date 1 .

At time zero, consumers can also trade in bonds, which are assets in zero net supply. The (after-tax) asset matrix is $\bar{W}=\left(W_{1}, W_{2}\right)$, where

$$
\begin{aligned}
& W_{1}=\left[\cdots\left(\begin{array}{c}
q^{j} \\
\left(1+t_{s}^{1}\right) R_{s}^{j}
\end{array}\right)_{j \in \mathcal{J}_{1}} \cdots\right]_{\left(N \times J_{1}\right)}, \\
& W_{2}=\left[\cdots\left(\begin{array}{c}
y_{0}^{j}-q^{j} \\
M^{j}\left(y_{1}, t^{1}\right)
\end{array}\right)_{j \in \mathcal{J}_{2}} \ldots\right]_{\left(N \times J_{2}\right)}
\end{aligned}
$$

and $M^{j}\left(y_{1}, t^{1}\right)$ has typical $s$-element $m_{s}^{j}=\left(1+t_{s}^{1}\right) y_{s}^{j}$. Lastly, agents face the same financial structure $\bar{W}$. Thus, they all have equal access opportunities to the equity market. Bankruptcy or default is not allowed.

\subsubsection{The consumer problem For notational simplicity, let}

$$
\widetilde{e}^{h}=\left(e_{0}^{h}+\sum_{j \in \mathcal{J}_{2}} q^{j} \bar{\theta}_{j}^{h}, e_{\mathbf{1}}^{h}+t_{\mathbf{1}}^{0}\right)
$$

define the total after-tax endowment of a typical consumer $h$. The individual budget set, for given financial opportunities $\bar{W}$ and total endowments $\widetilde{e}^{h}$, is

$$
\mathbb{B}\left(\bar{W}, \widetilde{e}^{h}\right)=\left\{x^{h} \in \mathbb{R}_{+}^{N}: \begin{array}{l}
\theta_{j}^{h} \geq 0, j \in \mathcal{J}_{2} \\
x^{h}-\widetilde{e}^{h}=\bar{W} \theta^{h}
\end{array}\right\} .
$$

An individual optimum for $h$, at $\left(\bar{W}, \widetilde{e}^{h}\right)$, is a pair $\left(\widehat{x}^{h}, \widehat{\theta}^{h}\right)$ such that $\widehat{x}^{h}$ maximizes $u^{h}\left(x^{h}\right)$ on $\mathbb{B}\left(\bar{W}, \widetilde{e}^{h}\right)$, and $\widehat{\theta}^{h}$ satisfies $\widehat{x}^{h}-\widetilde{e}^{h}=\bar{W} \widehat{\theta}^{h}$, and $\theta_{j}^{h} \geq 0$ for all $j$ in $\mathcal{J}_{2}$.

At an interior individual optimum, $\widehat{x}^{h} \gg 0$, the present value vector (or state price) of consumer $h$ in state $s$ is $\widehat{\lambda}_{s}^{h}=\lambda_{s}^{h}\left(\widehat{x}^{h}\right)=D_{x_{s}} u\left(\widehat{x}^{h}\right) / D_{x_{0}} u\left(\widehat{x}^{h}\right)$. Let $\langle\bar{W}\rangle$ denote the column span of $\bar{W}$, a subspace of dimension (at most) equal to $J$. Its orthogonal complement, $\langle\bar{W}\rangle^{\perp}$, has (at least) dimension $N-J$. If we assume, for the moment, that the no-short sale constraints are nonbinding, $\widehat{\lambda}^{h} \in\langle\bar{W}\rangle^{\perp}$.

2.1.5. The firm's problem If we keep on abstracting from the no-short sale constraints, the fact that, at the individual optimum, present value vectors satisfy $\lambda^{h} \in\langle\bar{W}\rangle^{\perp}$, implies that consumers do not typically agree on the evaluation of future income profiles. Since consumers are also firms' shareholders, the latter poses problems for the definition of the objective function of the firm. Precisely, if the firm chooses its production plans in the best 
interest of shareholders, none of its feasible choices will, typically, achieve shareholders' unanimous approval. ${ }^{3}$

Let $\beta^{j} \in \mathbb{R}_{++}^{N}$ be the evaluation criterion (or state price vector) given to firm $j \in \mathcal{J}_{2}$. An individual optimum of firm $j$ is a vector $y^{j}$ that maximizes $\beta^{j} y^{j}$ on $Y^{j}$. Next, assume that firm $j$ acts in the best interest of shareholders, and let $\beta^{j}$ be a (linear) function of its shareholders' present value vectors, $\lambda^{h}$. In principle, we would (at least) like to require that, at equilibrium, $\beta^{j}$ is consistent with the market value of the firm, $q^{j}$, where by consistent we mean that $q^{j}=\left(1 / \beta_{0}^{j}\right) \beta^{j} y^{j}$ (see De Marzo (1988)). Consumers' first-order conditions imply that the firm problem is well defined if $\left(1 / \beta_{0}^{j}\right) \beta^{j}$ belongs to $\langle\bar{W}\rangle^{\perp}$ (i.e., to the same subspace to which consumers' present value vectors belong). ${ }^{4}$ Then, any consistent $\beta^{j}$ leads to a well defined firm's problem.

Two consistent criteria, $\beta^{j}$, have been extensively used in the literature. One was proposed by Drèze, and the second by Grossman and Hart. In Drèze (1987) each firm takes production decisions in the best interest of final shareholders, by evaluating future profits with respect to $\beta^{j}=\sum_{h \in H} \theta_{j}^{h} \lambda^{h}$. In Grossman and Hart (1979), firms act in the best interest of initial (or date 0 ) shareholders, and $\beta^{j}=\sum_{h \in H} \bar{\theta}_{j}^{h} \lambda^{h}$. The two criteria mainly differ in the "timing" at which the evaluation of production projects is made. In Grossman and Hart projects are chosen when asset markets are still open. Thus shareholders may always "vote" against a production choice by selling their shares in the firm. At equilibrium, the no-arbitrage condition ensures that the market price they are paid matches their private evaluations. On the contrary, Drèze's criterion is based on the idea that production plans are decided after the security markets close. Thus, current (or final) shareholders rely entirely on the fact that production plans are decided in their interest. ${ }^{5}$

3 If financial markets are (generically) complete $(\operatorname{dim}\langle\bar{W}\rangle=J=S)$, consumers agree on project evaluations: $\lambda^{h}=\lambda \in\langle\bar{W}\rangle^{\perp}$ for all $h$, and $\operatorname{dim}\langle\bar{W}\rangle^{\perp}=1$. Thus, if we assume that firms act in the best interest of shareholders, it must be that, for given $\lambda$, a production plan $y^{j}$ is chosen such that $y^{j} \in \arg \max \left\{\lambda y^{j}: y^{j} \in Y^{j}\right\}$ for all $j$. By introducing this objective function, it is straightforward to show that the corresponding allocation is Pareto optimal. This objective function is also appropriate in the case of partial spanning. Partial spanning occurs when financial markets are incomplete, but firms are constrained to propose projects, whose returns exclusively lie in the span of the marketed securities.

4 A further necessary condition, that is more related to the fact that individual demands are well behaved, is that, in every state $s$, (at least) one consumer, $h(s)$, has non-satiated preferences. This ensures that, in equilibrium, $\lambda^{h(s)}(x)>0$, and thus that all firms are valued, preventing the security matrix from dropping rank.

5 Both criteria can be shown to be shareholder constrained efficient if lump-sum transfers among shareholders are allowed. Grossman-Hart's does also require that shareholders have competitive price perceptions (i.e., shareholders of firm $j$ correctly anticipate the effect of a change of the production plan of $j$, on the market value of the firm). Magill and Shafer (1991), p. 1586, discuss firms' constrained efficient pricing criteria. For completeness, we say that a decision $y^{j}$ of $j$ is shareholder constrained efficient if there does not exist a marginal change 
It will be clearer as we go along in the paper that our main results hold for any criterion, $\beta$, which is expressed as a functional of consumers' present value vectors, $\lambda$. For reasons of analytic tractability, we stick with the adoption of a specific criterion, and choose the following modified GrossmanHart criterion. ${ }^{6}$

Definition 2 (Modified Grossman-Hart criterion). For every $\left(\bar{\theta}_{j}, \lambda, t_{j}^{1}\right)$ and all $s \in \mathcal{S} \cup\{0\}, \beta_{s}^{j}=\sum_{h} \bar{\theta}_{j}^{h} \lambda_{s}^{h}\left(1+t_{s}^{1}\right)$ is the pricing criterion of firm $j \in \mathcal{J}_{2}$.

\subsection{The equilibrium with taxes}

Gathering the definitions of agents' actions and markets we obtain a full description of our economy, $\mathcal{E}(u, f, \eta, e, \bar{\theta}, R)$. We provide the following definition of equilibrium at the policy instrument vector $t^{I}$ in $T^{I}$.

Let $\omega=(\eta, e, \bar{\theta}, u, f)$, and let $\mathcal{E}$ be the set of economies with typical element $(\omega, R)$.

Definition 3 ( $E-t^{I}$ equilibrium). In an economy $(\omega, R)$ in $\mathcal{E}$, an equilibrium with tax instruments $t^{I} \in T^{I}$, and firm pricing criteria $\beta$, is a pair $((x, \theta, y),(q, t))$ such that:

(i) $\forall h,\left(x^{h}, \theta^{h}\right)$ is an individual optimum for consumer $h$, at $(q, y, \omega, t, R)$;

(ii) $\forall j, y^{j}$ is an individual optimum for firm $j$, at $\beta^{j}$;

(iii) $\forall j \in \mathcal{J}_{1}, \sum_{h} \theta_{j}^{h}=0 ; \forall j \in \mathcal{J}_{2}, \sum_{h} \theta_{j}^{h}=1$;

(iv) $t$ satisfies fiscal budget balance at $t^{I}$.

Observe that, if (i)-(iv) are satisfied, spot markets clear. Moreover, (iv) can be written differently depending on the type of fiscal policy assumed. For the equilibrium to be well defined every $\beta^{j}$ must be chosen in the set of consistent criteria (see Section 2.1.5). Finally, we define a no-tax equilibrium, an $E-t^{I}$ equilibrium setting all tax instruments $t^{I}$ equal to zero. Observe that our notion of no-tax equilibrium is the one-commodity analogue of the equilibrium notion in Geanakoplos et al. (1990), when firms' pricing criteria, $\beta$, are defined as in Drèze (1987); it is the one-commodity

$d y^{j}$ such that $y^{j}+d y^{j}$ is optimal for $j$ and $\left(1, \nabla u^{h}\right) \cdot \frac{\partial x^{h}}{\partial y^{j}} d y^{j} \geq 0$ for all shareholders $h$, with strict inequality for some $h$. Further, we say that $\beta^{j}$ is shareholder constrained efficient for $j$ if, at equilibrium, it supports a decision profile $y^{j}$ that is shareholder constrained efficient for $j$. Assume that lump-sum redistribution is possible at date zero. Then, $\beta^{j}$ is shareholder constrained efficient if and only if $\sum_{h}\left(1, \nabla u^{h}\right) \cdot\left(\partial x^{h} / \partial y^{j}\right) d y^{j} \leq 0$ for every $d y^{j}$ such that $\nabla f^{j} \cdot d y^{j}=0$.

6 This criterion can be shown to be shareholder constrained efficient if: (1) lump-sum transfers among shareholders are allowed, and (2) shareholders have competitive price perceptions (see the previous footnote). 
analogue of the equilibrium notion in Grossman-Hart (1979), when $\beta$ is the Grossman-Hart criterion; it coincides with a standard notion of pure exchange GEI equilibrium when $\mathcal{J}_{2}=\emptyset$.

\section{Welfare effects of tax reforms}

\subsection{Our main results}

Definition 4 (Income Tax-Constrained Pareto Optimality, IT-CPO). In every economy an $E-t^{I}$ equilibrium allocation of commodities fails to be IT-CPO if there exists a $t^{I^{\prime}}$ in $T^{I}$ such that the $E-t^{I^{\prime}}$ equilibrium allocation is Pareto superior.

For $(u, f, R)$ fixed, let $\Psi$ denote the set of economies, whose typical element $\Psi$ is denoted $(e, \eta, \bar{\theta})$. The set $\Omega=\Psi \times \mathrm{U} \times \mathrm{Y}$, with typical element $\omega=(\psi, u, f)$, is the set of economies, parametrized by $\psi$ together with the preferences and technologies. We are now ready to state our first result.

Theorem 1. Let Assumptions 1 and 2 hold, and $2 \leq H \leq S-J, J \geq 1$. There exists a generic set of economies $\Omega^{* *}$ in the parameter space $\Omega$ such that, for every $\omega$ in $\Omega^{* *}$, each no-tax, $E-t^{I}$ equilibrium is not an IT-CPO.

Observe that our theorem is stated and applies to both pure exchange economies and production economies (with or without bonds). As we show in Section 4 below, the requirement to prove Theorem 1 is that there is at least one asset $(J \geq 1)$; this can be either a bond or a stock.

To establish our main result we assume that the initial state coincides with a no-tax equilibrium $\left(E-t^{I}\right.$ with $\left.t^{I}=0\right)$. Further, we restrict attention to those economies, parameterized by initial endowments, for which notax equilibria exist and are regular (see Theorem 2, and Corollary 2, in Section 4). For fixed $(u, f, R)$, the set of regular economies is denoted by $\Psi^{*}$. Regularity of equilibria is necessary to carry out local analysis (or comparative statics), since it ensures (local) differentiability of consumer demand for commodities and assets, and (local) differentiability of the netsupply schedules. Moreover, we use the notation $\Omega^{*}=\Psi^{*} \times \mathrm{U} \times \mathrm{Y}$ the set of regular economies parameterized by endowments, preferences and technologies. For every $\omega \in \Omega^{*}$ a regular no-tax $E-t^{I}$ equilibrium exists; that is, for $t^{I}$ in an open neighborhood of zero, an $E-t^{I}$ equilibrium with $t^{I} \neq 0$ exists (again, see Theorem 2 and Corollary 2).

Referring to Section 4 for a complete proof of Theorem 1, here, we provide a sketch of its main arguments. Let $\xi=\left(\xi^{\prime}, t^{I}\right) \in \mathbb{R}^{n^{0}} \times T^{I}$ be a vector of endogenous variables and policy instruments appearing in the 
extended system of equilibrium first-order conditions, and let $F(\xi ; \omega)=0$ denote such a system (see Section 4 for the precise definitions). The number of equations in the system is $n$, equal to the sum of the number $n^{\prime}$ of endogenous variables and the number $S$ of policy instruments. The no-tax equilibrium set is

$$
E=\left\{(\xi, \omega): F(\xi ; \omega)=0, t^{I}=0\right\},
$$

and, when restricted to $\Omega^{*}$, it is a manifold. Next, define the function $V$ : $E \rightarrow \mathbb{R}^{H}$ such that $V(\xi ; \omega)=\left(u^{1}\left(x^{1}\right), \ldots, u^{H}\left(x^{H}\right)\right)$ is the vector of utility functions. Further, let

$$
G(\xi ; \omega)=\left[\begin{array}{c}
F(\xi ; \omega) \\
V(\xi ; \omega)
\end{array}\right] .
$$

To prove Theorem 1 it suffices to show that there exists a marginal tax change $d t^{I}$, in an open neighborhood of $t^{I}=0$, such that the new equilibrium $(\xi+d \xi)$ is Pareto superior, that is, $D_{\xi} V=\left(d u^{1}, \ldots, d u^{H}\right) \gg 0$. This can be done by proving that $G$ behaves locally as a submersion, i.e.,

$$
D G\left(\xi^{\prime}, t^{I} ; \omega\right)=\left(\begin{array}{l}
D F\left(\xi^{\prime}, t^{I} ; \omega\right) \\
D V\left(\xi^{\prime}, t^{I} ; \omega\right)
\end{array}\right)_{\left(\xi^{\prime}, t^{I} ; \omega\right) \in E}
$$

has full row rank. In fact, observe that, when the subspace spanned by the rows of $D G$ is equivalent to $\mathbb{R}^{n+H}$, it includes vectors whose first $n$ entries are zero and the remaining $H$ positive:

$$
\begin{aligned}
& D_{\xi^{\prime}} F d \xi^{\prime}+D_{t^{\prime \prime}} F d t^{I^{\prime \prime}}=0, \\
& D_{\xi^{\prime}} V d \xi^{\prime}+D_{t^{I \prime}} V d t^{I^{\prime \prime}}>0
\end{aligned}
$$

for some tax reform, $d t^{I}$, and corresponding changes in the endogenous equilibrium variables $d \xi^{\prime}$.7

An equivalent, but instructive, way to describe our proof is as follows. Assume that $G$ is a submersion at a no-tax equilibrium, for an economy $\omega$. Then, $r D G\left(\xi^{\prime}, t^{I}, \omega\right)=0$ if and only if $r=0$, i.e., the following system has no solution,

$$
\left(\begin{array}{c}
r D G\left(\xi^{\prime}, t^{I} ; \omega\right) \\
\|r\|-1
\end{array}\right)=0
$$

This has a natural interpretation in terms of standard welfare analysis. Assume that a planner chooses $\left(\xi^{\prime}, t^{I}\right)$ such that first-order (necessary) conditions for the optimality of the equilibrium allocation hold: the welfare function we have in mind is $\mathcal{W}=\sum_{h} a^{h} u^{h}\left(x^{h}\right)+\mu F\left(\xi^{\prime}, t^{I} ; \omega\right)$, with

7 Constrained suboptimality is equivalent to the existence of a feasible direction of tax reforms in the sense of Guesnerie (1977). 
multipliers $(a, \mu)$. Then, the above technique is used to show that $t^{I}=0$ would typically not satisfy the first-order (necessary) conditions for a local maximum of $\mathcal{W}$ with Lagrange multipliers $r=(\mu, a)$, except for the trivial case $r=0 .{ }^{8}$

In showing that (1) has no solution, we have to check that the number of policy objectives (the utility levels) do not exceed the number of instruments (tax/transfers). ${ }^{9}$ Then we prove that the (local) property of $G$ is generic: we show that our result holds for a set of economies, $\Omega^{* *}$, that is (nonempty) open and dense in $\Omega^{*}{ }^{10}$

Observe that the submersion property of $G$ is a sufficient condition for proving the statement of Theorem 1. If $D G$ is locally onto, then the subspace spanned by the rows of $D G$ is equivalent to $\mathbb{R}^{n+H}$, therefore including vectors whose first $n$ entries are zero with the remaining $H$ either positive or negative. This implies that there also exist directions $d t^{I^{\prime \prime}}$ which are socially undesirable,

$$
\begin{aligned}
& D_{\xi^{\prime}} F d \xi^{\prime}+D_{t^{I \prime}} F d t^{I^{\prime}}=0, \\
& D_{\xi^{\prime}} V d \xi^{\prime}+D_{t^{I \prime}} V d t^{I^{\prime}} \leq 0 .
\end{aligned}
$$

This directly implies the following.

Corollary 1. In the context of Theorem 1, for almost every regular economy $\omega$ in $\Omega^{* *}$, there exist tax reforms which can achieve any direction of utility changes in an open neighborhood of the no-tax equilibrium allocations.

This generalization of Theorem 1 should be read as a warning to policy makers. The effects of our income tax reforms on private risk-sharing opportunities may be socially undesirable. This should even increase the level of concern if interpreted in the context of our simple economy. In contrast

${ }^{8}$ Here, one has to be a little careful. The latter planner's problem is compact but fails to be convex. The non-convexity of the set of centralized attainable allocations implies that the constrained Pareto frontier need not be convex either. Then, the set of maxima obtained from the Lagrangian fails to provide a complete representation of the constrained Pareto frontier (e.g., any concave segment of the frontier is not attainable from the Lagrangian maximization). Yet, the proof of our main result shows that the first-order conditions of the above problem, evaluated at the no-tax equilibrium, do typically fail to hold. Therefore, competitive equilibria are, typically, not extrema (neither maxima nor minima nor saddle points) of that problem, and $r=0$.

9 Observe that the number of equations in the new system $r D G=0$ is equal to the number of columns of $D G$, and thus to the number of original unknowns $\left(z^{\prime}, t\right)$. The unknowns of $r D G=0$ are the components of $r$ whose number is equal to the number of equations in $G=0$.

10 These steps follow the submersion approach, originally due to Smale (1974). Geanakoplos and Polemarchakis (1986) first suggested its application to prove constrained suboptimality of equilibria when asset markets are incomplete. 
with most of the GEI literature, our results are not driven by changes in relative spot prices (as, for example, in Geanakoplos and Polemarchakis (1986), Geanakoplos et al. (1990), Cass and Citanna (1998), Citanna et al. (1998)). Yet, relative price changes would certainly increase the level of complexity of any policy analysis, by introducing an additional policy transmission channel. Another potential source of complexity is the policy scheme. Here, we have used a very simplified definition of constrained suboptimality, with very strong restrictions on the type of policy interventions allowed to the planner: interventions are anonymous; publicly announced when all markets are still open (i.e., they are also ex-post constrained efficient); fiscal budget is required to balance in every date and state of the world (see Definition 1). All these assumptions, if changed, would increase the complexity of equilibrium computations, and policy evaluations.

Remark 1 (Extending Theorem 1 to $E-t^{I}$ with $t^{I} \neq 0$ ). Our main result holds when the initial state is a regular equilibrium with a non-trivial tax system, $t^{I}$. Although we do not provide a complete argument in support of our claim, the reader can check that this is true by adapting the proof of Theorem 1 below.

\subsection{Concluding comments}

Our results contribute to highlighting the welfare properties of personal income taxation in incomplete market economies. As pointed out in the introduction, we focus on a particular aspect of state-contingent taxation, namely, its ability to affect private risk-sharing opportunities through direct asset span effects. A local marginal change of the tax system acts directly on the asset span, changing (but not augmenting) consumers' ability to redistribute income across contingencies. Thus, even if the tax reforms considered do not reduce the degree of market incompleteness, they may modify individual risk-sharing opportunities, allowing consumers to reallocate resources in a direction that was initially financially unfeasible. When this happens, we may say that the tax reform has social insurance effects.

Before going further, recall that the use of tax reforms entails a different constrained optimality criterion with respect to the one traditionally used in the GEI literature which we refer to as Diamond constrained efficiency (or simply CPO; see Diamond (1967), Geanakoplos and Polemarchakis (1986), and Geanakoplos et al. (1990)). Thus, to ease the interpretation of our results, a comparison of the welfare properties of equilibria under the two constrained optimality criteria may help the reader. Moreover, since the definitions of the set of CPO allocations, and the welfare properties of equilibria, are different in a pure exchange economy in comparison with a production economy, we shall keep the two cases distinct. Again, this is possible since, 
as we show in Section 4 below, Theorem 1 holds for a class of economies that encompasses these two.

Pure exchange GEI. In a pure exchange economy, a CPO is an allocation that cannot be Pareto improved by a portfolio redistribution, when date zero lump-sum transfers are also a viable policy instrument. It is well-known that, in this setting, every competitive GEI entails a CPO allocation. Instead, Theorem 1 shows that there exists a generic set of economies in which every pure exchange equilibrium is not an IT-CPO. In other words, our notion of constrained efficiency (Definition 4) is stronger than the one proposed by Diamond.

Example 1. Consider an economy with one firm, two consumers, and two states of uncertainty ( $J=J_{1}=1, H=S=2$ ). Consumers have different, quasi-linear, utilities, and identical endowments:

$$
\begin{aligned}
& u^{1}\left(x^{1}\right)=x_{0}^{1}+5 \sqrt{x_{1}^{1}}+x_{2}^{1}, \\
& u^{2}\left(x^{2}\right)=x_{0}^{2}+x_{1}^{2}+5 \sqrt{x_{2}^{2}}, \\
& e^{1}=e^{2}=(3, .5, .5) .
\end{aligned}
$$

The only asset is a riskless bond $R=(1,1)$. This economy has a unique equilibrium that coincides with the no-trade equilibrium. Figure 1(a) represents the equilibrium, in the space of second-period consumption, with the thick lines being the present value budget constraints (evaluated at the equilibrium individual state prices), and the dotted line representing the income transfers line (i.e., the asset span).

- An optimal tax reform. The equilibrium can be locally Pareto improved via a marginal income tax reform: $d t^{1}=(-1,-5) \%$; we are taxing the return from the asset in state 2 more heavily than in state 1 . Consumer 1 , who has a particularly strong taste for consumption in state 1, buys the bond issued by consumer 2, who instead has relatively stronger taste for the good in state 2 . The sacrifice of 2, in terms of second-period consumption, is compensated by an increase in her consumption at date zero, due to her gains in asset trade. The summary of results is expressed in percentage change from the bench mark no-tax CPO equilibrium:

\begin{tabular}{rrrrrr}
\hline & $\% \Delta x_{0}$ & $\% \Delta x_{1}$ & $\% \Delta x_{2}$ & $\% \Delta \theta$ & $\% \Delta u$ \\
\hline$h=1$ & -6.71 & 1.5 & 1.45 & 1.52 & .04 \\
$h=2$ & 6.71 & -1.5 & -1.45 & -1.52 & .04 \\
\hline
\end{tabular}

Figure 1(a) represents the equilibrium in the space of second-period income (i.e., for given equilibrium levels of first-period consumption). The tax reform tilts the asset span-line clockwise (see Fig. 1(b)); at the new 


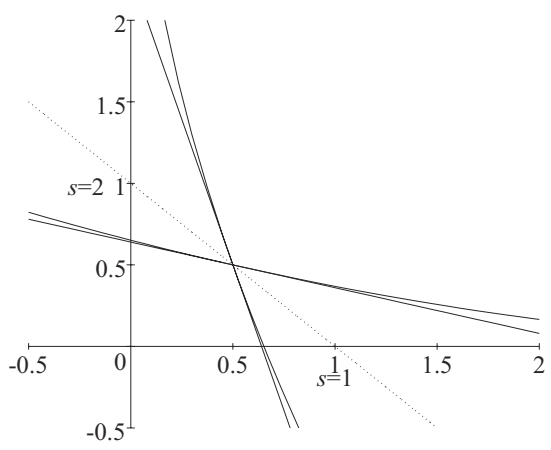

(a)

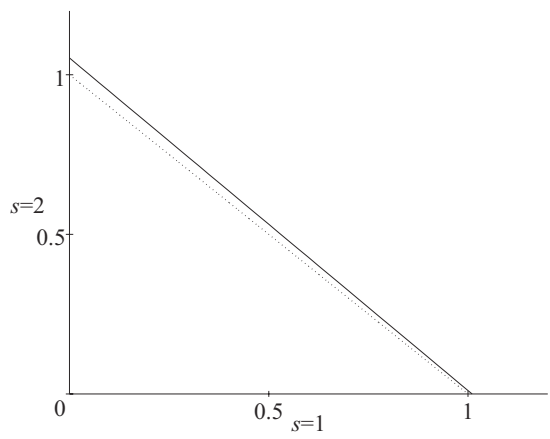

(b)

Fig. 1.

equilibrium state prices, the present value budget constraints of 1 and 2 , respectively, become flatter and steeper, to support the new allocations.

Production GEI. In a production economy, a CPO is a consumption and production allocation that cannot be Pareto improved by any alternative resource-feasible allocation, which is achievable through (i) portfolio redistributions, (ii) (technologically feasible) changes of production decisions, (iii) date zero, lump-sum transfers. In other words, centralized income allocations are still implemented via portfolio transfers; yet, in comparison with a pure exchange, the planner can affect the asset-payoff structure through (technologically feasible) production decisions.

Ray technologies. In the context of a one-commodity GEI, Diamond showed that equilibria are CPO, provided that multi-linear (or ray) production technologies are assumed (see Diamond (1967)). If more general forms of technology are introduced, equilibria need not be CPO. This was first demonstrated by an example of Drèze (see Drèze (1987), Chapter 4, p. 278) and, more recently, subjected to further investigation in Dierker et al. (1999). Yet, two things should be noted. First, even with ray technologies a CPO equilibrium need not be an IT-CPO. State-contingent tax reforms may modify production plans in a way that is not technologically feasible, by independently controlling production output, state-by-state (although not firm-by-firm). An illustration of our claim can be immediately derived from Example 1, by assuming that the only asset is a production plan, $y_{s}=\mu_{s} y_{0}, \mu_{s} \in \mathbb{R}_{+}$, for all $s>0$. Then, tax reforms allow the planner to control $y_{s}$, independently across states, a much more powerful intervention than just setting $y_{0}$.

General production technologies. One can show that CPO equilibria may fail to be IT-CPO for more general forms of technologies. Let us consider the examples in Drèze (1987) and Dierker et al. (1999). First, recall that the type of argument provided in these examples is not based on marginal 
reforms; instead, discrete changes in portfolios and/or production allocations are required to show constrained inefficiency. Both examples do, in fact, exploit the nonconvexity of the set of centralized attainable allocations and show that equilibrium allocations may fail to be globally efficient. For instance, in Dierker et al. (1999) the only interior ${ }^{11}$, Drèze, equilibrium is at the minimum aggregate welfare point; yet this is a local CPO equilibrium: a marginal production change, and a portfolio readjustment, may locally Pareto improve the allocation, but cannot be supported as a competitive equilibrium. To put it differently, although the tax reform may be Pareto improving, it may not survive a successive round of competitive trade. The only two other equilibria are corners, in which the only firm in the economy is, respectively, owned by the first and by the second consumer. This example is a robust case in which an interior Drèze equilibrium is a local (but not global) CPO equilibrium. In Example 2 below, first, we show that the interior equilibrium, despite being a local CPO, is not an IT-CPO; second, we show that even the two corner equilibria, which are global CPO, fail to be IT-CPO. To put it differently, tax reforms (as in Definition 1) may have firstorder effects that are strong enough to achieve a local Pareto improvement, while the same cannot be said of policy reforms in the sense of Diamond.

Example 2. Consider an economy with utilities as in Example 1 above, in which consumers are partners of the only firm,

$$
Y=\left\{y \in \mathbb{R}^{3}: y_{0}=-1, y_{1}+y_{2} \leq-y_{0}\right\} .
$$

This economy has three (no-tax) Dréze equilibria: a symmetric equilibrium with each consumer holding $1 / 2$ of the firm producing $y=(-1,0.5,0.5)$, and two corner equilibria in which consumer 1 (respectively, consumer 2) is the single owner of the firm and $y=(-1,1,0)$ (resp., $y=(-1,0,1)$ ) is produced. Figure 2(a) below represents equilibria in the space of secondperiod consumption (where, for clarity, we omit present value budget constraints). ${ }^{12}$ Equilibria can be perfectly Pareto ranked: assuming identical welfare weights, and allowing for date zero lump-sum transfers, the symmetric equilibrium is Pareto dominated by either of the two alternative corner equilibria; the latter two, instead, have the same welfare properties. Moreover, since all equilibria are technologically efficient, this ranking can be thought to depend only on their risk sharing properties. Corner equilibria are global CPO (the symmetric equilibrium is a local, but not a global, CPO

\footnotetext{
11 Here we use the term "interior" referring both to portfolios and allocation: an interior equilibrium is one in which two (or more) consumers are partners in each firm, and such that individual consumption is strictly positive.

12 At the first corner equilibrium $\left(\theta_{1}=y_{1}=1.0\right), \beta_{2}=\lambda_{2}^{1} \leq \beta_{1}=\lambda_{1}^{1}$ guarantees that the production plan is a profit maximizer at $\beta$. The second corner equilibrium is symmetric to the first.
} 
equilibrium). In Fig. 2(b) below, we plot the aggregate (indirect) utility level as a function of the return from holding the firm in state 1 (i.e., $\theta^{1} y_{1}$ ), and fixed $x_{0}$, when allocations are feasible in the sense of Diamond. It is immediate that the minimum welfare is obtained at the symmetric allocation, while the only two CPO are corner solutions. Thus, Fig. 2(b) shows that at a minimum welfare point, the symmetric equilibrium is a local CPOequilibrium: a Pareto superior equilibrium can be achieved by a Diamond central planner via discrete portfolios and production reallocations.

- Optimal tax reforms 1. (Interior equilibrium) We start from the interior equilibrium, and assume that a tax reform, such as the one considered in Example 1, is introduced: $d t^{1}=(-1,-5) \%$. Then a Pareto improvement is obtained with a $.04 \%$ increase in the utility of both agents, and an $4.17 \%$ increase in the share of agent 1 in the firm:

\begin{tabular}{rrrrrr}
\hline & $\% \Delta x_{0}$ & $\% \Delta x_{1}$ & $\% \Delta x_{2}$ & $\% \Delta \theta$ & $\% \Delta u$ \\
\hline$h=1$ & -7.86 & 1.06 & 1.98 & 4.17 & .04 \\
$h=2$ & 7.86 & -1.06 & -1.98 & -4.17 & .04 \\
\hline
\end{tabular}

2. (Corner equilibria) Since the two corner equilibria are symmetric, we consider only the one in which consumer 1 is the single owner of the firm. The tax reform $d t^{1}=(.1,0) \%$ is weakly Pareto improving:

\begin{tabular}{rrrrrr}
\hline & $\% \Delta x_{0}$ & $\% \Delta x_{1}$ & $\% \Delta x_{2}$ & $\% \Delta \theta$ & $\% \Delta u$ \\
\hline$h=1$ & -.05 & 0.5 & 0 & 0 & .06 \\
$h=2$ & 0.5 & -0.5 & 0 & 0 & 0 \\
\hline
\end{tabular}

In words, a (global) CPO equilibrium can be improved upon by using proportional income taxes.

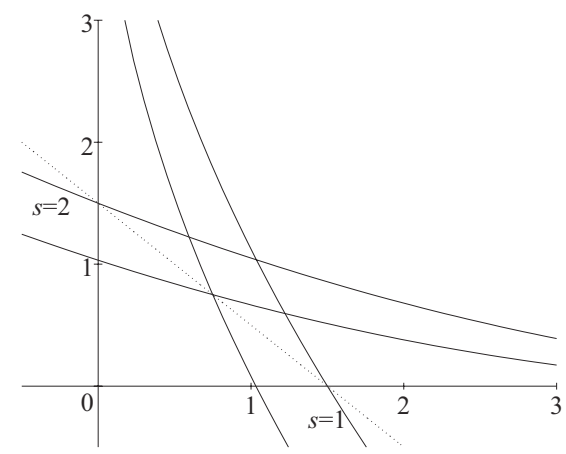

(a)

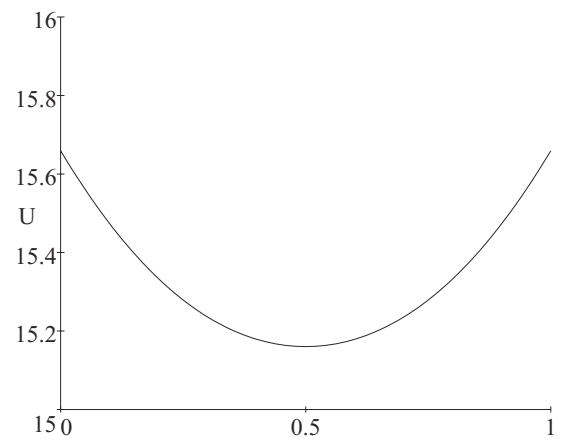

(b)

Fig. 2. 
Theorem 1 establishes a set of sufficient conditions for marginal, personal income tax reforms to provide social insurance. In particular, the following requirements must be met: markets are sufficiently incomplete, and consumers sufficiently heterogeneous, $2 \leq H \leq S-J$; there are enough policy instruments; the initial economy is chosen in a generic set in which interiority conditions hold (see Theorem 2 in Section 4). The special case of a representative consumer, who is also the single owner of the only firm in the economy $(H=J=1)$, is analyzed in Diamond and Mirrlees (1992). Diamond and Mirrlees show that there exist economies where ad valorem contingent taxes on dividends can be used to implement a first best equilibrium. ${ }^{13}$ Yet, even in this simple case the type of economy for which optimality is achieved is that peculiar type in which consumers do not want to redistribute income across states. In other words, economies in which the initial endowment distribution, or the type of consumer preference, is such that the restrictions arising from market incompleteness to consumers are non-binding. ${ }^{14}$

For a better understanding of our results, we go back to our economy. Local perturbations of the tax system act directly on the asset span, tilting the subspace of feasible income transfers away from its original position. In doing so, the consumer may transfer income in a direction that is orthogonal to the initial asset span. Yet, unlike in the case of fixed payoff structures, ${ }^{15}$ or in that of simple linear technologies (e.g., Example 2), the final asset span crucially depends on firms' behavior. It is far from obvious that production decisions do not offset the effects of tax reform, thereby neutralizing the planner's policy. ${ }^{16}$ To complete our analysis of Theorem 1 we need to bring into the picture production decisions and firms' state prices, $\beta$. Our explanation of Theorem 1 is based on the idea that firms, unlike the central planner, take the asset span as given. First, observe that, at $t^{1}=0$, firm $j$ 's pricing criterion reduces to $\beta^{j, 0}=\sum_{h} \theta_{j}^{h} \lambda^{h}$; hence, the initial no-tax equilibrium allocation is still optimal for firm $j$ if the tax reform does not have a direct

13 I thank Herakles Polemarchakis for pointing out this reference to me.

14 A further peculiar situation is represented by the case of generically (or effectively) complete markets. When the number of consumer types satisfies $1 \leq H \leq J$, it is always possible to find asset structures that would allow consumers to effectively achieve a full efficient diversification of market risk.

15 See also the literature on real indeterminacy (e.g., Geanakoplos and Mas-Colell (1989), Balasko and Cass (1989)).

16 Remember that after each policy reform re-allocations of commodity and assets are completely decentralised (i.e., achieved competitively by private agents through trade on the existent commodity and asset markets). 
asset span effect: the matrix

$$
\left[\begin{array}{lll}
d t_{1}^{1} & & \\
& \ddots & \\
& & d t_{S}^{1}
\end{array}\right] M\left(y_{1}, 0\right) .
$$

induces the same column span of $M\left(y_{1}, 0\right) .{ }^{17}$ Theorem 1 shows that, if this is not the case, $\left\langle M\left(y_{1}, 0\right)\right\rangle \neq\left\langle M\left(y_{1}, d t^{1}\right)\right\rangle$, then the initial production decisions are no longer individually optimal at $\beta^{j, 0}\left(\beta^{j, 0}\left(d t^{1} \square y^{j}\right) \neq 0\right)$ for some $d t^{1}$ and some $j .{ }^{18}$ Moreover, there is room for a change of the (after tax) payoff vectors in a direction that was otherwise unfeasible for the private sector at the initial, no-tax, equilibrium $\left(d t^{1} \square y^{j} \in\left\langle M\left(y_{1}, 0\right)\right\rangle^{\perp}\right) .{ }^{19}$ Yet, for production changes not to kill the direct span effect of a tax reform, we need to account for indirect (or equilibrium) state-price effects (i.e., pecuniary externalities), that is, changes in $\beta^{j}$ which may support the new allocation as an individual optimum for $j .{ }^{20}$ Our proof provides direct calculations confirming this intuition. It also shows that the assumption that the planner computes the equilibrium, accounting for the state-price externalities, is not essential: the argument in the proof also goes through if the central planner does not anticipate the effects of a change in individual state prices $(\lambda)$ on firms' state prices $(\beta)$. Thus, no particular informational advantage is required for the planner to implement a tax reform. For our theorem to hold, the planner is "only" required to have statistical information on the consumer types, firm technologies, state prices, and market structure. That is, based on a complete, but abstract, representation of the economy, a Pareto improving tax reform can be designed by computing and comparing different equilibria. Once a tax reform has been chosen, and the corresponding tax vector publicly announced, the final allocation is determined competitively, with each agent truthfully revealing her/his type.

17 In fact, a tax reform characterized by uniform tax changes across states satisfies $\beta^{j, 0}\left(d t^{1} \square y^{j}+d y^{j}\right)=0$, and thus $\beta^{j, 0}\left(d t^{1} \square y^{j}\right)=0$, for every individually optimal initial production allocation $y^{j}$ (i.e., $d y^{j}$ such that $\nabla f^{j}\left(y^{j}\right) \cdot d y^{j} \leq 0$ ), that is, the initial no-tax production plans are individually optimal. Here, and elsewhere, $\square$ denotes the elementwise vector product: for $x, y \in \mathbb{R}^{C}, x \square y=\left(x_{1} y_{1}, \ldots, x_{C} y_{C}\right)^{\prime}$.

18 A tax reform that produces a direct span effect implies that $d t^{1} \square y^{j} \notin\left\langle M\left(0, y_{1}\right)\right\rangle$. Then, $\beta^{j, 0}\left(d t^{1} \square y^{j}+d y^{j}\right) \neq 0$ for every individually optimal initial production allocation $y^{j}$ (i.e., $d y^{j}$ such that $D f^{j}\left(y^{j}\right) \cdot d y^{j}=0$ ). This implies that $y^{j}$ need not be ex-post optimal for $j$ at $\beta^{j, 0}$.

19 Here, by "feasibility" we are directly referring to financial feasibility. On technological feasibility, observe that after tax-payoffs of a firm $j,\left(1+d t^{1}\right) \square y^{j}$, may not be in $Y^{j}$.

20 At the tax equilibrium, arising after a tax reform, the production plan $y^{j^{\prime}}=y^{j}+d y^{j}$ is optimal for $j$ at $\beta^{j}=\beta^{j, 0}+d \beta^{j, 0}$ if $D f^{j}\left(y^{j \prime}\right)$ is collinear to $\beta^{j}$, that is, if the production choice tilts the gradient of the transformation function away from the original position. 
It should be clear, by now, that our focus on "capital income taxes" is made without loss of generality. Our analysis and all our results extend substantially unchanged to either of the following alternative tax policies:

(a) a proportional, state-contingent, anonymous, tax/subsidy system on individual total income (returns from portfolio holdings plus endowment income);

(b) a proportional, state-contingent, asset (or sector) specific, tax/subsidy system on individual capital income.

In (a), if the two components of individual income can be taxed independently, no further instrument is required to achieve state-by-state fiscal budget balance. On the contrary, if income is subject to a state-contingent uniform tax a second policy variable is needed in each state to achieve within states-budget balance; a variable that does not introduce any further distortion is a state-contingent, anonymous, lump-sum transfer as the one considered in Definition 1. This last observation applies to (b), where up to $J$ policy variables can be used in each state. Yet, now such an increase in the number of tax variables implies the loss of anonymity (taxes/subsidies become firm-type specific). ${ }^{21}$ If bonds are taxed, (b) accounts for the case in which interest payments are tax deductible, as well as for the case in which they are not.

Remark 2 (Asset span versus relative spot price effects). A marginal tax reform, $d t^{I} \in \mathbb{R}^{S}$, induces a first-order welfare effect on (the indirect utility of) $h$ that, in a multi-commodity GEI, is equivalent to:

(i) the second-order effect (pecuniary externality) produced via a change of relative spot prices by a planner acting under the CPO criterion as in Geanakoplos and Polemarchakis (1986) and Geanakoplos et al. (1990);

(ii) the second-order effect (pecuniary externality) produced by the taxation of trades in assets in Citanna et al. (2001).

Indeed, in an exchange GEI, a marginal change in tax instruments has the following key effect on the utility of $h$ (see Equation 8 in Section 4):

$$
\begin{aligned}
d u^{h} & =\lambda^{h} Z^{h} D_{t^{I}} M d t^{I} \\
& =\left(\lambda_{1}^{h} z_{1}^{h}, \ldots, \lambda_{S}^{h} z_{S}^{h}\right)\left(\begin{array}{cccc}
d t_{1}^{I} & & \\
& \ddots & \\
& & d t_{S}^{I}
\end{array}\right) M d t^{I} .
\end{aligned}
$$

In an exchange GEI with multiple commodities, a similar effect is obtained through changes in relative spot prices: when $d q=0, d u^{h}=\lambda^{h} Z^{h} D_{\zeta} p d \zeta$,

21 A fiscal policy like (b) has been analyzed in Tirelli (1999). 
for a policy reform $d \zeta$ (e.g., see Equation (71) in Magill and Shafer (1991), and Section 3 in Citanna et al. (2001)).

If $H \leq S-J$, our main result shows that there exists a generic set of economies in which equilibria possess sufficient utility variation (see (iv) in Theorem 2 below). This upper bound condition is only sufficient, as we have shown in Examples 1, 2 above.

\section{Proofs}

First-order (sufficient) conditions (Foc's) for the existence of an interior $E-t^{I}$ (with $\left(x^{h}, \theta_{j}^{h}, y^{j}\right) \gg 0$ for all $j \in \mathcal{J}_{2}, \theta_{j}^{h} \neq 0$ for all $j \in \mathcal{J}_{1}$ and all $h)$ can be written as:
(1) $\forall h$,
$D u^{h}\left(x^{h}\right)-\lambda^{h}=0$
NH
(2) $\forall h$,
$-x^{h}+\widetilde{e}^{h}+W\left(q, y, t^{1}\right) \theta^{h}=0$
$\mathrm{NH}$
(3) $\forall h$,
$\lambda^{h} W\left(q, y, t^{1}\right)=0$
$J H$
(4) $\forall j$,
$\beta^{j}-v_{j} D f^{j}\left(y^{j}\right)=0$,
$\beta^{j}=\sum_{h} \bar{\theta}_{j}^{h} \lambda^{h} \square\left(1+t^{1}\right)$
(5) $\forall j$,
$f^{j}\left(y^{j}\right)=0$
$J_{2}$
(6) $\forall j \in \mathcal{J}_{1}, \sum_{h} \theta_{j}^{h}=0$
$\forall j \in \mathcal{J}_{2}, \quad \sum_{h} \theta_{j}^{h}-1=0$
(7) $\forall s$,$$
\sum_{h \in \mathcal{H}}^{h}\left(t_{s}^{0}+t_{s}^{1}\left(\sum_{j \in \mathcal{J}_{1}} \theta_{j}^{h} R_{s}^{j}+\sum_{j \in \mathcal{J}_{2}} \theta_{j}^{h} y_{s}^{j}\right)\right)=0,
$$

where $\lambda, v$ are Lagrange multipliers. ${ }^{22}$ Each subsystem of equations is indexed by Arabic numbers on the left-hand side, and its dimension is indicated on the right-hand side.

Let $\widetilde{F}: \Xi^{\prime} \times T^{I} \times \Psi \rightarrow \mathbb{R}^{n^{\prime}}$, where $\widetilde{F}\left(\xi^{\prime}, t^{I}, \psi\right)=0$ represents the left-hand side of the system of equilibrium first-order conditions, excluding the government budget constraint, where $\Xi^{\prime} \subset \mathbb{R}^{n^{\prime}}$ has typical element $\xi^{\prime}=\left(x, \theta, \lambda, y, v, q, t^{0}\right)$, with $n^{\prime}=2 H N+H J+J N+2 J+S$, and $\Psi \subset \mathbb{R}_{+}^{k}$ denotes the parameter space, with typical element $\psi=(e, \eta, \bar{\theta})$, and $k=N(H+J)+H J$. Here, without loss of generality, we let the policy

\footnotetext{
22 In particular, the first two multipliers are attached to the constraints of the consumer problem ( $\rho$ refers to the no-short sale constraints). Here $v$ is the vector of multipliers related to the firms' optimization problem.
} 
instrument vector be $t^{I}=t^{1}$. Adding the government budget constraints to $\widetilde{F}$, we obtain a new system of equations which we denote by $F: \Xi \times \Psi \rightarrow$ $\mathbb{R}^{n}$, with $n=n^{\prime}+S$ and $\Xi=\Xi^{\prime} \times T^{I} \subset \mathbb{R}^{n^{\prime}+S}$. Thus $F=0$ represents the complete system of first-order conditions of the $E-t^{I}$.

We endow the space of functions $\mathrm{Y} \times \mathrm{U}$ with the $\mathcal{C}^{2}$ compact-open (weak) topology. For simplicity, let $\mathrm{Y} \times \mathrm{U}$ denote the topological space $\mathrm{Y} \times \mathrm{U}, \mathcal{C}^{2}(\mathrm{Y}) \times \mathcal{C}^{2}(\mathrm{U})$. We also assume that the space of endowments has the usual (Euclidean) topology.

We are now ready to establish local existence of an interior equilibrium.

Theorem 2 (Local existence and regularity of an $E-t^{I}, t^{I}=0$ ). Consider an economy $\mathcal{E}(\omega)$ with fixed $\left(u^{h}, f^{j}\right)_{h, j}$. Further, let Assumptions 1,2 hold and $H \leq S-J$. If $t^{I}=0$, there exists a set of economies $\Psi^{*} \subset \Psi$, open and of full measure, such that, for every $\psi \in \Psi^{*}$, there exists a (locally isolated) $E-t^{I}$ equilibrium with the following properties:

(i) the equilibrium is regular;

(ii) $\bar{W}$ has full rank;

(iii) $\theta_{j}^{h} \neq 0$ for all $h, j$;

(iv) the following matrix has full rank $H$ :

$$
\Lambda Z=\left(\begin{array}{ccc}
\lambda_{1}^{1}\left(z_{1}^{1}-\bar{y}_{1} / H\right) & \cdots & \lambda_{S}^{1}\left(z_{S}^{1}-\bar{y}_{S} / H\right) \\
\vdots & & \vdots \\
\lambda_{1}^{H}\left(z_{1}^{H}-\bar{y}_{1} / H\right) & \cdots & \lambda_{S}^{H}\left(z_{S}^{H}-\bar{y}_{S} / H\right)
\end{array}\right),
$$

where $z=x-e, \bar{y}_{s}=\sum_{j \in \mathcal{J}_{2}} y_{s}^{j}$.

Proof. Generic existence and regularity of $E-t^{I}$ at $t^{I}=0$ substantially follow from Theorem 2 in Geanakoplos et al. (1990). Generic properties (i) through (iii) are standard ${ }^{23,24}$ while (iv) coincides with the property in item 3 of Lemma 1 of Citanna et al. (2001) in a pure exchange GEI $\left(J_{1}=0\right)$. To

23 Geanakoplos et al. $(1986,1989,1990)$ point out that there are two sources of nondifferentiability of security demand functions $\left(\theta_{j}^{h}\right)$ which follow from the no-short sales assumption. The first occurs whenever $\theta_{j}^{h}=0, \rho_{j}^{h}=0$ (i.e., the constraint is binding, but this has "no cost" for the consumer). Yet, one can show that such a case in not generic in the endowments. The most problematic source of non-differentiability occurs when $\theta_{j}^{h}=0$ for some $(h, j)$ and $\operatorname{rank}(W)<J$. However, assuming that $(H+J) \leq N$, they show that: (i) there exists a generic set of economies for which equilibria are of full rank; (ii) in restricting attention to such economies, typically equilibria are such that the no-short sale constraints do not bind. The latter implies that $\theta_{j}^{h} \geq 0$ for all $h$ and all $j \in \mathcal{J}_{2}$.

24 To show regularity we can endow the parameter space, $\Psi$, with the usual topology: let $\mathcal{O} \subset \mathbb{R}^{N(H+J)} \times \mathbb{R}_{+}^{H J}$ represent the class of (non-empty and) open subsets of $\mathbb{R}^{N(H+J)} \times$ $\mathbb{R}_{+}^{H J}$; a subset $\hat{\Psi} \subset \Psi$ is open if and only if $\hat{\Psi}=O \cap \Psi$ for some $O \in \mathcal{O}$. 
show that this property holds we can proceed by appending

$$
\begin{aligned}
\left(b_{1}, \ldots, b_{H}\right) \wedge Z & =0, \\
b b^{T}-1 & =0
\end{aligned}
$$

to the equilibrium system, $F=0$, and show that the new system as no solution at $t^{I}=0$ (for this we need that $H \leq S-J$ ). This property is implied by the well-known property of local controllability of individual state prices, $\lambda$. Thus, restrict attention to the set $\Psi^{*}$ for which Theorem 2 in Geanakoplos et al. (1990) holds, and (i) through (iv) are satisfied; these properties can be shown to hold generically in consumers' endowments, $e$. Observe that, at $t^{I}=0, \widetilde{F}$ and the system of first-order GEI equilibrium conditions, $F$, are identical, and that so are their Jacobians with respect to $\xi^{\prime}$. Therefore,

$$
\operatorname{rank}\left(\left.D_{\xi^{\prime}} \widetilde{F}\left(\xi^{\prime}, t^{I}, \psi\right)\right|_{\widetilde{F}\left(\xi^{\prime}, t^{I}, \psi\right)=0}\right)_{t^{I}=0}=n^{\prime},
$$

and the set of regular equilibria, $\widetilde{F}^{-1}(0)$, is a manifold of dimension $k=$ $\operatorname{dim}(\Psi)$. Next, we add the government budget constraint to $\widetilde{F}$ to get $F$, the complete system of first-order conditions of the $E-t^{I}$ equilibrium. We want to show that the Jacobian of $F$, evaluated at a no-tax equilibrium, has full rank. Now $\left(D_{\xi^{\prime}, t^{I}} F \mid \widetilde{F}\left(\xi^{\prime}, t^{I}, \psi\right)=0\right)_{t^{I}=0}$ has the following structure (its complete representation is in (5) below, with the last column and row blocks deleted):

$$
\begin{aligned}
& \text { var. } \\
& \text { equ. } \\
& \widetilde{F} \\
& \left.\sum_{h \in \mathcal{H}}\left(t_{s}^{0}+t_{s}^{1}\left(\sum_{j \in \mathcal{J}_{1}} \theta_{j}^{h} R^{j}+\sum_{j \in \mathcal{J}_{2}} \theta_{j}^{h} y_{1}^{j}\right)\right)=0 \mid \begin{array}{ccc}
n^{\prime} \times n^{\prime} & 0 & {\left[\sum_{\substack{j \in \mathcal{J}_{2} \\
S \times S}} y_{s}^{j}\right.}
\end{array}\right]^{(*)}
\end{aligned}
$$



$(*):\left[\xi_{s}\right]$ denotes a block-diagonal matrix with typical element $\xi_{s}$.

Since we take $\psi \in \Psi^{*}$, we have that $\sum_{j} y_{s}^{j} \neq 0$ for all $s$. Thus, if 0 is a regular value of $\widetilde{F}$, it is also a regular value of $F$.

By straightforward application of the implicit function theorem, we obtain the following.

Corollary 2. In the context of Theorem 2 , for every $\psi \in \Psi^{*}$, there exists an open neighborhood $\mathcal{N}(0)$ of $t^{I}=0$ such that, for all $t^{I} \in \mathcal{N}(0)$,

$$
\operatorname{rank}\left(\left.D_{\xi^{\prime}, t^{I}} F\left(\xi^{\prime}, t^{I}, \psi\right)\right|_{F\left(\xi^{\prime}, t^{I}, \psi\right)=0}\right)=n,
$$

and $F\left(\xi^{\prime}, t^{I}, \psi\right)$ is transverse to zero. 
Next, restricting attention to economies in $\Psi^{*}$, we turn to proving Theorem 1 . We do so by showing that $G=\left(F, u^{1}, \ldots, u^{H}\right)\left(\xi^{\prime}, t^{I}, \omega\right)$ behaves as a submersion, when it is evaluated at a no-tax equilibrium of $\psi \in \Psi^{*}$. As we have already argued, this is equivalent to showing that the following system (locally) has no solution:

$$
0=\left[\begin{array}{c}
r D_{\xi} G \\
\|r\|-1
\end{array}\right]_{\left(\xi^{\prime}, t^{I}, \omega\right) \in E} .
$$

To this end, it is sufficient to show that there exists a local parameterization of the economy such that (4) has the desired (local) properties. For reasons that will soon become clear, the type of parameterization that does the job is one that allows us to perturb the Hessian of $u$ and $f$ without altering the values of the functions and their gradients. The latter is convenient, since it implies that perturbations do not modify the equilibrium first-order conditions. One such class of functions is the one that admits quadratic perturbations. Denoting by $\operatorname{cl} \mathcal{N}$ the closure of the $\operatorname{set} \mathcal{N}$, let

$$
\begin{aligned}
u^{h}\left(x^{h} ; A^{h}\right) & =u^{h}\left(x^{h}\right)+\frac{1}{2}\left(x^{h}-\hat{x}^{h}\right)^{T} A^{h} \gamma^{h}\left(x^{h}\right)\left(x^{h}-\hat{x}^{h}\right) \text { if } x^{h} \in \mathcal{N}\left(\hat{x}^{h}\right) \\
& =u^{h}\left(x^{h}\right) \text { if } x^{h} \notin \operatorname{cl} \mathcal{N}_{\epsilon}\left(\hat{x}^{h}\right), \\
\mathrm{f}^{j}\left(y^{j} ; B^{j}\right) & =f^{j}\left(y^{j}\right)+\frac{1}{2}\left(y^{j}-\hat{y}^{j}\right)^{T} B^{j} \delta^{j}\left(y^{j}\right)\left(y^{j}-\hat{y}^{j}\right) \text { if } y^{j} \in \mathcal{N}\left(\hat{y}^{j}\right) \\
& =f^{j}\left(y^{j}\right) \text { if } y^{j} \notin \operatorname{clN} \mathcal{N}_{\epsilon}\left(\hat{y}^{j}\right) .
\end{aligned}
$$

Here $A^{h}$ is a square symmetric $N$-dimensional matrix of parameters, which are taken to be sufficiently small to preserve strict quasi-concavity of the utility function. Define by $\mathcal{N}$, and $\mathcal{N}_{\epsilon}$, neighborhoods of $\hat{x}^{h}$, possibly empty, such that $\operatorname{cl} \mathcal{N} \subset \mathcal{N}_{\epsilon} \subset \operatorname{cl} \mathcal{N}_{\epsilon} \subset \mathbb{R}_{++}^{S}, \epsilon>0$. Further, $\gamma^{h}$ are smooth bump functions (see Hirsch (1976), p. 41), taking value 1 if $x^{h} \in \mathcal{N}$, and 0 if $x^{h} \notin \operatorname{cl} \mathcal{N}_{\epsilon}$. The same construction has been used for $\mathrm{f}^{j}$.

Without loss of generality, the Jacobian of $G$ with respect to $\left(\xi^{\prime}, t^{1}\right)$, evaluated at $t^{I}=t^{1}=0$, is represented in the following table with the first column referring to the equation numbering in (2) above, and the first row reporting the variables with respect to which derivatives are computed. ${ }^{25}$

25 Observe that delating the last row block from the above Jacobian provides the representation of the Jacobian of the equilibrium first-order conditions of an $E-t^{I}$ (i.e., the Jacobian of $F$ ), evaluated at $t^{I}=t^{1}=0$. 


\begin{tabular}{|c|c|c|c|c|c|c|c|c|}
\hline & $\begin{array}{l}\ldots x^{h} \ldots \\
---\end{array}$ & $\begin{array}{c}\ldots \theta^{h} \ldots \\
----\end{array}$ & $\begin{array}{c}\ldots \lambda^{h} \ldots \\
----\end{array}$ & $\begin{array}{l}\ldots v^{j} \ldots \\
---\end{array}$ & $\begin{array}{c}\ldots y^{j} \ldots \\
----\end{array}$ & $\begin{array}{c}\ldots q^{j} \ldots \\
----\end{array}$ & $\begin{array}{c}t^{1} \\
-----\end{array}$ & $\begin{array}{c}t^{0} \\
--\end{array}$ \\
\hline & {$\left[D^{2} u^{h}\right]$} & 0 & $-\left[I_{N}\right]$ & 0 & 0 & 0 & 0 & 0 \\
\hline 2 & $-I_{N H}$ & {$[W]$} & 0 & 0 & $\ldots \theta_{j}^{h} I_{N} \ldots$ & $\begin{array}{c}\bar{\theta}^{h^{T}}-\theta^{h^{T}} \\
0_{S \times J}\end{array}$ & $\begin{array}{c}0, \ldots, 0 \\
{\left[\sum_{j} \theta_{j}^{h} y_{s}^{j}\right]}\end{array}$ & $\begin{array}{c}0 \\
I_{S}\end{array}$ \\
\hline 3 & 0 & 0 & {$\left[W^{T}\right]$} & 0 & {$\left[\lambda^{h}\right]$} & $\lambda_{0}^{h} I_{J}$ & $\begin{array}{c}\ldots, 0, \ldots \\
\ldots, \lambda_{s}^{h} y_{s}, \ldots\end{array}$ & 0 \\
\hline 4 & 0 & 0 & $\begin{array}{c}\ldots \bar{\theta}_{j}^{h} I_{N} \ldots \\
\ldots \ldots \ldots \ldots\end{array}$ & {$\left[D f^{j^{T}}\right]$} & $-\left[D^{2} f^{j}\right]$ & 0 & $\begin{array}{c}0, \ldots, 0 \\
\sum_{h} \bar{\theta}_{j}^{h}\left[\lambda_{s}^{h}\right]\end{array}$ & 0 \\
\hline 5 & 0 & 0 & 0 & 0 & {$\left[D f^{j}\right]$} & 0 & 0 & 0 \\
\hline 6 & 0 & $\ldots I_{J} \ldots$ & 0 & 0 & 0 & 0 & 0 & 0 \\
\hline 7 & 0 & 0 & 0 & 0 & 0 & 0 & {$\left[\sum_{j \in \mathcal{T}} y_{s}^{j}\right]$} & $H I_{S}$ \\
\hline 8 & {$\left[D u^{h}\right]$} & 0 & 0 & 0 & 0 & 0 & 0 & 0 \\
\hline
\end{tabular}

$\left[c_{s}\right]$ denotes a diagonal matrix with typical diagonal element $c_{s}$ varying across $s$.

Looking at Equation (2), it is immediate that, if $D G$ has full row rank, $r D G=0$ has equations outnumbering the unknowns $r=\left(r^{1}, \ldots, r^{8}\right) \in$ $\mathbb{R}^{n+H}$. Thus, it is sufficient to show that the Jacobian (5) has independent rows. An explicit formulation of (4) is:

\begin{tabular}{llll} 
(I) & $\forall h, r_{h}^{1} D^{2} \mathrm{u}^{h}-r_{h}^{2}+r_{h}^{8} D u^{h}=0$ & $N H$ & $r^{1}$ \\
(II) & $\forall h, j, r_{h}^{2} W^{j}+r_{j}^{6}=0$ & $J H$ & $r^{3}$ \\
(III) & $\forall h,-r_{h}^{1}+r_{h}^{3} W^{T}+\sum_{j} \bar{\theta}_{j}^{h} r_{j}^{4}=0$ & $N H$ & $r^{2}$ \\
(IV) & $\forall j \in \mathcal{J}_{2}, r_{j}^{4} D f^{j^{T}}=0$ & $J_{2}$ & $r^{5}$ \\
(V) & $\forall s, \forall j \in \mathcal{J}_{2},-\sum_{h}\left(\theta_{j}^{h} r_{h, s}^{2}-r_{h, j}^{3} \lambda_{s}^{h}\right)$ & $N J_{2}$ & $r^{4}$ \\
\multicolumn{5}{c}{$-r_{j}^{4} D_{. s}^{2} \mathrm{f}-r_{j}^{5} D_{s} f^{j}=0$} & & \\
(VI) & $\forall j, \sum_{h} r_{h, 0}^{2}\left(\theta_{j}^{h}-\bar{\theta}_{j}^{h}\right)-\sum_{h} \lambda_{0}^{h} r_{h j}^{3}=0$ & $J$ & $r^{6}$ \\
(VII) & $\forall s \in \mathcal{S}, \sum_{j, h}\left(\theta_{j}^{h} r_{h, s}^{2} W_{s}^{j}+r_{h, j}^{3} \lambda_{s}^{h} W_{s}^{j}\right)$ & $S$ & $\left(r^{8}\right)$ \\
& $\quad+\sum_{j \in \mathcal{J}_{2}}\left(r_{s}^{7} W_{s}^{j}+r_{j, s}^{4} \beta_{s}^{j}\right)=0$ & & \\
(VIII) & $\forall s \in \mathcal{S}, \sum_{h} r_{h, s}^{2}+H r_{s}^{7}=0$ & $S$ & $r^{7}$ \\
(IX) & $\|r\|-1=0$ & 1 &
\end{tabular}

where each block of equations is labelled in Roman numbers on the left-hand side, and the last two columns on the right-hand side indicate the number of equations in each block, with the unknown variables $(r)$ to which they can be matched. 
Proof of Theorem 1. Let $\Omega^{*}$ be a subset of $\Omega$ restricted to $\Psi^{*}$, and $\Omega^{* *}$ be a subset of $\Omega^{*}$ such that, for every $\omega \in \Omega^{* *}, G(\omega)$ is a submersion. Applying Lemma 1 and 2 below we conclude that $\Omega^{* *}$ is open and dense in $\Omega$.

Lemma 1. $\Omega^{* *} \subset \Omega^{*}$ is open.

Proof. Recall that $\xi=\left(\xi^{\prime}, t^{1}\right)$, and let $D_{\xi} V^{\alpha}$ denote a generic submatrix of the Jacobian of $V$ with respect to $\xi$. Define

$$
K_{\alpha}=\left\{u \in \mathbb{R}^{H}: \operatorname{det}\left(D_{\xi} V^{\alpha}\right)=0\right\}=\operatorname{det}\left(D_{\xi} V^{\alpha}\right)^{-1}(0) .
$$

This set is closed in $\mathbb{R}^{H}{ }^{26}$ The set of critical points of the map $V, N=$ $\left\{u \in \mathbb{R}^{H}: \operatorname{det}\left(D_{\xi} V^{\alpha}\right)=0 \quad \forall \alpha\right\}=\cap_{\alpha} K_{\alpha}$, is also closed. Therefore its complement $N_{R}=\mathbb{R}^{H} / N$ is open.

Let the function $\varphi: E \rightarrow \Omega$ be the natural projection of the equilibrium manifold onto the parameter space. Define

$$
B_{c}=\left\{(\xi, u, f, \psi) \in E:\left[\begin{array}{c}
\operatorname{det}\left(D_{\xi} F\right) \\
\operatorname{det}\left(D_{\xi} V^{\alpha}\right)
\end{array}\right]=0 \quad \forall \alpha\right\},
$$

the set of critical values of $\varphi . B_{c}$ is relatively closed, being the preimage of $\{0\}$ under a continuous function. Then, to ensure that the image of $B_{c}$ under the mapping $\varphi$ is closed, it suffices to establish that $\varphi$ is proper, which is proved as Lemma 3 in the appendix. Thus, $\varphi\left(B_{c}\right)$ is closed in $\Omega^{*}$, and its complement, $\Omega^{* *}=\Omega^{*} \backslash \varphi\left(B_{c}\right)$, is relatively open.

Lemma 2. $\Omega^{* *}$ is dense.

Proof. We divide the proof into two parts: the first considers a pure exchange economy, $\mathcal{J}_{1} \neq \emptyset, \mathcal{J}_{2}=\emptyset$, while the second deals with a production economy, $\mathcal{J}_{2} \neq \emptyset$.

Part 1 (pure exchange GEI) $\mathcal{J}_{1} \neq \emptyset, \mathcal{J}_{2}=\emptyset$.

In the case of a pure exchange, $\mathcal{J}_{2}=\emptyset, J=J_{1}$, (6) reduces to:

$$
\begin{array}{llll}
\text { (I) } & \forall h, r_{h}^{1}\left(D^{2} u^{h}+A^{h}\right)-r_{h}^{2}+r_{h}^{8} D u^{h}=0 & H N & r^{1} \\
\text { (II) } & \forall h, r_{h}^{2} W+r^{6}=0 & H J & r^{3} \\
\text { (III) } & \forall h,-r_{h}^{1}+r_{h}^{3} W^{T}=0 & H N & r^{2} \\
\text { (VI) } & \forall j, \sum_{h} r_{h 0}^{2} \theta_{j}^{h}-\sum_{h} \lambda_{0}^{h} r_{h j}^{3}=0 & J & r^{6} \\
\text { (VII) } & \forall s \in \mathcal{S}, \sum_{h, j}\left(\theta_{j}^{h} r_{h, s}^{2}+r_{h, j}^{3} \lambda_{s}^{h}\right) R_{s}^{j}=0 & S & \left(r^{8}\right) \\
\text { (VIII) } & \forall s \in \mathcal{S}, \sum_{h} r_{h, s}^{2}+H r_{s}^{7}=0 & S & r^{7} \\
\text { (IX) } & \|r\|-1=0 & 1 . &
\end{array}
$$$$
\text { (IX) } \quad\|r\|-1=0
$$

26 This is because it is the preimage of a singleton by a continuous function, in a complete metric space. Here, the continuous function is the polynomial associated to the determinant of the continuous linear mappings $D_{z} V^{\alpha \prime} s$. 
Matching the equations in system (7) with the unknowns it is immediate that if $H \leq S$ the system has equations outnumbering the unknowns $(r){ }^{27}$ Therefore, we are left to show that system (7) has independent equations. The proof can be divided into the following two cases (a) and (b).

Case (a): $\quad r_{h}^{1} \neq 0$ for all $h$

The following perturbations can be used: $\left(\left(d r_{., s}^{2}\right)_{s=1}^{J}, d r^{1}\right)$, respectively, for Equations (II), (III); $d r_{h}^{3}$ for $h \neq H$, for (VI); $d r^{7}$ for (VIII); $d A^{h}$ for $(I)_{h}$ (given $r_{h}^{1} \neq 0$ ) for all $h$. Finally, use $\left(\left(d r_{H, s}^{2}\right)_{s>J}, d r_{H}^{3}\right)$ for (VII).

Case (b): $\quad r_{h}^{1}=0$ for some $h$

Since, as we are going to show, $r_{h}^{1}=0$, for some $h$, implies that $r_{h}^{1}=0$ for all $h$, we cannot use quadratic perturbations of utilities in the present case. Yet, our result continues to hold when $r^{1}=0$, and the $u^{h}$ are fixed in $\mathrm{U}$.

Consider system (7). Now $r_{h}^{1}=0$, say for consumer $h=H$, implies that $r_{H}^{2}-r_{H}^{8} D u^{H}=0$. Then, consumer $H$ 's foc's and (II) imply $r^{6}=0$. Since $r^{6}$ is independent of $h, r_{h}^{2} \in\langle W\rangle^{\perp}$ holds for all $h$ such that $r_{h}^{8} \neq 0$. By (III), $r_{h}^{1}=W r_{h}^{3^{T}}$, i.e., either $r_{h}^{1} \in\langle W\rangle$ or $r_{h}^{3}=0$ (and $r_{h}^{1}=0$ ) for all $h \neq H$. Then, post-multiplying $(\mathrm{I})_{h}$ by $r_{h}^{1}$, and using the latter result, together with strict quasi-concavity of $u^{h}$, we have $r_{h}^{1}=0$. This holds for all $h$.

Also $r^{1}=0$ implies $r^{3}=0$ by (III). Then, consumers' foc's $\left(\lambda^{h} \cdot R^{j}=q_{j}\right)$ imply that we can rewrite (VII) as $\sum_{h} r_{h}^{8} \lambda_{s}^{h} \sum_{j} R_{s}^{j} \theta_{j}^{h}=\sum_{h} r_{h}^{8} \lambda_{s}^{h} z_{s}^{h}$ for all $s \in \mathcal{S}$, or, using the notation introduced in Theorem 2 , as

$$
\left(r_{1}^{8}, \ldots, r_{H}^{8}\right) \Lambda Z=0,
$$

where $\Lambda Z$ in (3) reduces to

$$
\Lambda Z=\left(\begin{array}{ccc}
\lambda_{1}^{1} z_{1}^{1} & \cdots & \lambda_{S}^{1} z_{S}^{1} \\
\vdots & & \vdots \\
\lambda_{1}^{H} z_{1}^{H} & \cdots & \lambda_{S}^{H} z_{S}^{H}
\end{array}\right) .
$$

Since we are considering economies in $\Psi^{*}$, under the assumption that $H \leq S-J$, Theorem 2 implies that $r^{8}=\left(r_{1}^{8}, \ldots, r_{H}^{8}\right)=0$. This immediately implies $r=0$. System (7) has no solution.

Part 2 (production GEI) $\mathcal{J}_{2} \neq \emptyset$.

We prove the result for the case of a pure production economy (with $\left.\mathcal{J}_{1}=\emptyset, \mathcal{J}_{2} \neq \emptyset\right)$, since then extending the argument to mixed cases is

27 This has a natural interpretation: the number of policy objectives (the utility levels) should not exceed the number of tax instruments (contingent taxes/transfers). The latter condition is an incomplete market analogue of the one due to Tinbergen (1952). Thus, for example, this bound could be dropped by introducing date 0 , lump-sum transfers, $\tau \in \mathbb{R}^{H}$. 
straightforward. As for Part 1, the proof reduces to showing that system (6) has independent equations.

\section{Case (a): $\quad r_{h}^{1} \neq 0$ for all $h$}

This is analogous to Case (a) in Part 1, except for Equations (IV), (V), which can, respectively, be perturbed by $d r_{0}^{4},\left(\left(d r_{., S}^{4}\right)_{s>0}, d r^{5}\right)$.

\section{Case (b): $\quad r_{h}^{1}=0$ for some $h$}

If we allow $r_{h}^{1}=0\left(r_{j}^{4}=0\right)$ for some $h$ (respectively, $j$ ), we cannot use perturbations of utility (resp., transformation) functions for those $h$ (resp., $j)$. We proceed, first, by showing that $r_{h}^{1} \neq 0, r_{j}^{4} \neq 0$ for (at least) one pair $(h, j)$, implies that system (6) has no solution (Fact 1). Then, in Fact 2, we prove that all other cases have the same implication.

Fact 1. If $r_{h}^{1} \neq 0, r_{j}^{4} \neq 0$ for, say, $(h, j)=(H, J)$, then system (6) has no solution.

Now $r_{H}^{1} \neq 0, r_{J}^{4} \neq 0$ imply that we can certainly use perturbations of utility and transformation functions for $H$ and $J$, respectively. Consider the "worst" possible case, in which $r_{h}^{1}=0$ for all $h \neq H$. Since $r_{h}^{1}=0$ implies $r_{h}^{2}=r_{h}^{8} D u^{h}$, by $(\mathrm{I})_{h}$, Equation (II) $)_{h}$ becomes redundant when the system is evaluated at equilibrium. Moreover, we can substitute for $r_{h}^{2}$ using (I) ${ }_{h}$ to drop this equation from (6). Then, the following perturbations can be used in the new system: $\left(\left(d r_{H, s}^{2}\right)_{s=1}^{J}, d r^{1}, d r_{., 0}^{4}\right)$, respectively, for Equations (II) $)_{H}$, (III), (IV); $d r_{h}^{3}$ for some $h \neq H$, for (VI); $d r^{7}$ for (VIII); $\left(\left(d r_{h}^{8}\right)_{h<H}, d r_{H, 0}^{2}\right)$ for (IX); $d A^{H}$ for (I) ${ }_{H}$ (given $r_{H}^{1} \neq 0$ ). Lastly, use $\left(\left(d r_{j, s}^{4}\right)_{s>0}, d r_{j}^{5}\right)$ for $(\mathrm{V})_{j}$ if $j \neq J$, and $d B^{j}$ otherwise; the latter implies that we can use $\left(d r_{J, s}^{4}\right)_{s>0}$ to perturb (VII).

Fact 2. Let $r_{h}^{1}=0$ for some $h$. Then, for $r \in\left\{r: r^{1}=0\right\} \cup\left\{r: r^{4}=0\right\}$, system (6) has no solution.

We divide our argument into four steps, (2.0)-(2.3).

(2.0) $\left(r^{1}, r^{4}\right)=0$ implies $r=0$ :

Firstly, $r_{h}^{1}=0$ implies $r_{h}^{2}=r_{h}^{8} D u^{h}$ by (I), and $r^{6}=0$ by (II) and consumers' foc's; also $r^{3}=0$ by (III). Then, consumers' foc's $\left(\lambda^{h} \cdot R^{j}=q_{j}\right)$ imply that we can rewrite (VII) as

$$
\left(r_{1}^{8}, \ldots, r_{H}^{8}\right)\left(\begin{array}{ccc}
\lambda_{1}^{1}\left(z_{1}^{1}-\bar{y}_{1} / H\right) & \cdots & \lambda_{S}^{1}\left(z_{S}^{1}-\bar{y}_{S} / H\right) \\
\vdots & & \vdots \\
\lambda_{1}^{H}\left(z_{1}^{H}-\bar{y}_{1} / H\right) & \cdots & \lambda_{S}^{H}\left(z_{S}^{H}-\bar{y}_{S} / H\right)
\end{array}\right)=0
$$


where $\bar{y}=\sum_{j} y^{j}$. Since we are considering economies in $\Psi^{*}$, under the assumption that $H \leq S-J$, Theorem 2 implies that $r^{8}=0$, and thus $r=0$. System (6) has no solution.

(2.1) $r_{h}^{1}=0$, for some $h$, implies that either $r_{j}^{4} \in\langle W\rangle^{\perp}$ or $r_{j}^{4}=0$ for all $j$ : Suppose not, so that $r_{j}^{4} \in\langle W\rangle /\{0\}$ for some $j$, and $r_{h}^{1}=0$ for some $h$; the latter implies $r_{h}^{2}=r_{h}^{8} D u^{h}$ by (I), and $r^{6}=0$ by (II) and consumers' foc's; next, postmultiplying $(\mathrm{V})_{j}$ by $r_{j}^{4}$, and using strict quasi-convexity of $f^{j}$, yields a contradiction, and so $r_{j}^{4}=0$.

(2.2) $r^{1}=0$ implies $r=0$ :

Now $r_{h}^{1}=0$ implies that $r_{j}^{4} \in\langle W\rangle^{\perp}$ or $r_{j}^{4}=0$ for all $j$, by (2.1). Also $r_{h}^{1}=0$ for all $h$, and the latter, implies $r_{h}^{3}=0$ for all $h$, by (III) (and the full rank property of $W$ ). Then, (III) and (I), respectively, imply $\sum_{j \in \mathcal{J}_{2}} \bar{\theta}_{j}^{h} r_{j, s}^{4}=0, r_{h, s}^{2}=r_{h}^{8} D_{s} u^{h}$ for all $h, s$. Using these results, and consumers' foc's $\left(\lambda^{h} \cdot R^{j}=q_{j}\right)$, in (VII) yields (9) in (2.0) above. Therefore, $r=0$.

(2.3) $r_{h}^{1}=0$ for some $h$ and $r^{4}=0$ implies $r=0$ :

Firstly, $r_{h}^{1}=0$, for some $h$, implies $r_{h}^{2}=r_{h}^{8} D u^{h}$ by (I), and $r^{6}=0$, $r_{h}^{2} W=0$ for all $h$, by (II). Using $r_{j}^{4}=0$, for all $j$, in (III) implies that either $r_{h}^{1}=r_{h}^{3} W^{T}$ or $\left(r_{h}^{3}, r_{h}^{1}\right)=0$ for all $h$. Postmultiplying (I) by $r_{h}^{1^{T}}$, and using the latter result, yields $r_{h}^{3} W^{T} D^{2} u^{h} W r_{h}^{3^{T}}=0$ for all $h$. By strict quasi-concavity of $u^{h}$, this holds iff $r_{h}^{3}=0$ for all $h$, and hence $r^{1}=0$. Finally, we can apply (2.0).

We can finally gather the results in Cases (a) and (b), respectively in Parts 1, 2 above, and conclude the proof by applying Sard's theorem: there exists a dense set of economies, $\Omega^{* *}$ in $\Omega^{*}$, such that, for every $\omega$ in $\Omega^{* *}$, $G(\omega)$ is a submersion (see, e.g., Guillemin and Pollack (1974), p. 62).

\section{Appendix}

Lemma 3. The natural projection $\varphi: E \rightarrow \Omega$ is proper.

Proof. Let $C$ be a compact and nonempty subset of $\Omega$. We have to show that, for each converging sequence $\left(\omega^{r}\right)_{r=1,2, \ldots},\left(\omega^{r}\right) \rightarrow \omega^{*}, \omega^{r}, \omega^{*} \in C$, the sequence $\varphi^{-1}\left(\omega^{r}\right)=\left(\xi^{r}, \omega^{r}\right)$ converges in $E$ (i.e., $\left(\xi^{r}, \omega^{r}\right) \rightarrow\left(\xi^{*}, \omega^{*}\right) \in$ $E)$. First, note that every parameter vector $\left(\omega^{r}\right) \in C$ has boundaries. Second, (by Assumption 2(2)) there are subsequences such that $y^{r}$ is defined on a compact set when $\omega^{r} \in C$; also, by continuity of $\mathrm{f}, \mathrm{f}\left(y^{r}\right) \rightarrow\left|\mathrm{f}\left(y^{*}\right)\right|<\infty$, i.e., (5) of the equilibrium first-order conditions (2) is satisfied. Further, since $\mathrm{f}$ is twice continuously differentiable, $D \mathrm{f}\left(y^{r}\right) \rightarrow\left|D \mathrm{f}\left(y^{*}\right)\right|<\infty$ 
(for any finite $B$ ). The asset market clearing condition, together with the no-short sell conditions, imply that $\theta^{r} \rightarrow\left|\theta^{*}\right|<\infty$ is a subsequence of $\left(\theta^{r}\right)$. Then, Assumption 2(2), and the spot market clearing conditions, imply that individual demands have subsequences $\left(x^{r}\right) \rightarrow\left|x^{*}\right|<\infty$ such that (2) holds. Since the utility function is twice continuously differentiable, $D u\left(x^{r}\right) \rightarrow\left|D u\left(x^{*}\right)\right|$ (for any finite $A$ ). This also implies that $\left(\lambda^{r}\right) \rightarrow$ $\left|\lambda^{*}\right|<\infty$, and thus (1) is satisfied. The last condition - together with the fact that $\theta^{r}$ is bounded - implies that $\beta^{r}$ also converges to a finite bounded entity. Then, note that, by definition of $T^{\prime},\left(t^{1 r}\right)$ is always bounded. The above facts imply that for every firm the Lagrange multiplier $v^{r}$ is such that $v^{r} \rightarrow\left|v^{*}\right|<\infty$ and (4) holds. Moreover, the above facts, and (3) and (7) respectively, also imply that there exist subsequences $q^{r} \rightarrow\left|q^{*}\right|<\infty$, and $\left(t^{0 r}\right) \rightarrow\left|t^{0}\right|<\infty$. This completes the proof.

\section{References}

Balasko, Y., Cass, D. (1989): The structure of financial equilibrium with exogenous yields: the case of incomplete markets. Econometrica 57, 135-162

Cass, D., Citanna, A. (1998): Pareto improving financial innovation in incomplete markets. Economic Theory 11, 467-494

Citanna, A., Kajii, A., Villanacci, A. (1998): Constrained suboptimality in incomplete markets: a general approach and two applications. Economic Theory 11, 495-521

Citanna, A., Polemarchakis, H.M., Tirelli, M. (2001): The taxation of trades in assets. Working Paper No. 01-21. Department of Economics, Brown University, Providence, RI

De Marzo, P. (1988): An extension of the Modigliani-Miller theorem to stochastic economies with incomplete markets and independent securities. Journal of Economic Theory $\mathbf{4 5}$, 353-369

Diamond, P.A. (1967): The role of a stock market in a general equilibrium model with technological uncertainty. American Economic Review 57, 759-776

Diamond, P.A., Mirrlees, J.A. (1992): Optimal taxation of identical consumers when markets are incomplete. In: Dasgupta, P. et al. (eds.): Economic analysis of markets and games: essays in honor of Frank Hahn. MIT Press, Cambridge, MA, pp. 561-581

Dierker, E., Dierker, H., Grodal, B. (1999): Incomplete markets and the firm. Paper 9902. Department of Economics, University of Vienna, Vienna

Drèze, J.H. (1987): Investment under private ownership: optimality, equilibrium and stability. In: Drèze, J.H.: Essays on economic decisions under uncertainty. Cambridge University Press, Cambridge, pp. 261-297

Elul, R. (1995): Welfare effects of financial innovation in incomplete markets economies with several consumption goods. Journal of Economic Theory 65, 43-78

Elul, R. (1999): Welfare-improving financial innovation with a single good. Economic Theory 13, 25-40

Feldstein, M. (1976): On the theory of tax reform. Journal of Public Economics 6, 77-104 
Geanakoplos, J., Magill, M., Quinzii, M., Drèze, J. (1990): Generic inefficiency of stock market equilibrium when markets are incomplete. Journal of Mathematical Economics 19, 113-151

Geanakoplos, J., Mas-Colell, A. (1989): Real indeterminacy with financial assets. Journal of Economic Theory 47, 22-38

Geanakoplos, J., Polemarchakis, H. (1986): Existence, regularity, and constrained suboptimality of competitive allocations when the asset market is incomplete. In: Heller, W.P., et al. (eds.): Uncertainty, information, and communication. Essays in honor of Kenneth J. Arrow. Vol. III. Cambridge University Press, Cambridge, pp. 65-95

Grossman, S.J., Hart, O.D. (1979): A theory of competitive equilibrium in stock market economies. Econometrica 47, 293-329

Guesnerie, R. (1977): On the direction of tax reforms. Journal of Public Economics 7, 179-202

Guillemin, V., Pollack, A. (1974): Differential topology. Prentice Hall, Englewood Cliffs, $\mathrm{NJ}$

Hirsch, M.W. (1976): Differential topology. Springer, New York

Magill, M., Shafer, W. (1991): Incomplete markets. In: Hildenbrand, W., Sonnenschein, H. (eds.): Handbook of mathematical economics. Vol. IV. North-Holland, Amsterdam, pp. $1523-1614$

Smale, S. (1974): Global analysis and economics. III. Pareto optima and price equilibria. Journal of Mathematical Economics 1, 107-117

Tinbergen, J. (1952): On the theory of economic policy. North-Holland, Amsterdam

Tirelli, M. (1999): Three essays on incomplete markets. Ph.D. dissertation. Columbia University, New York 